\title{
Towards a Natural Theory of Dark Energy: Supersymmetric Large Extra Dimensions ${ }^{1}$
}

\author{
C.P. Burgess \\ Physics Department, McGill University, Montréal, Québec, Canada. \\ Department of Physics and Astronomy, McMaster University, \\ Hamilton, Ontario, Canada. \\ Perimeter Institute, Waterloo, Ontario, Canada.
}

\begin{abstract}
The first part of this article summarizes the evidence for Dark Energy and Dark Matter, as well as the naturalness issues which plague current theories of Dark Energy. The main point of this part is to argue why these naturalness issues should provide the central theoretical guidance for the search for a successful theory. The second part of the article describes the present status of what I regard as being the best mechanism yet proposed for addressing this issue: Six-dimensional Supergravity with submillimetre-sized Extra Dimensions (Supersymmetric Large Extra Dimensions, or SLED for short). Besides summarizing the SLED proposal itself, this section also describes the tests which this model has passed, the main criticisms which have been raised, and the remaining challenges which remain to be checked. The bottom line is that the proposal survives the tests which have been completed to date, and predicts several distinctive experimental signatures for cosmology, tests of gravity and for accelerator-based particle physics.
\end{abstract}

\section{CONTENTS}

\section{DARK ENERGY}

(a) Dark Matter and Dark Energy: The Evidence: i. Dark Matter; ii. Dark Energy.

(b) Modelling Dark Energy: i. Scales; ii. Sensitivity to Initial Conditions.

(c) Naturalness Issues: i. The Problem of Quantum Corrections; ii. Two Categories of Fine Tuning; iii. 'Technical' Naturalness; iv. The Problem of 'Self Tuning': Weinberg's No-Go Theorem.

\section{SUPERSYMMETRIC LARGE EXTRA DIMENSIONS}

(a) The Proposal: i. The Action.

(b) Classical Relaxation of the 4D Cosmological Constant: i. $6 D$ Self-Tuning: A Cartoon; ii. $6 D$ Self-Tuning I: General Solutions; iii. 6D Self-Tuning II: Response to Tension Changes; iv. 6D Self-Tuning III: A 4D Analysis?

(c) Quantum Bulk Contributions: Why There is Something and Not Nothing: i. Bulk Loops I: UV Sensitivity; ii. Bulk Loops II: Weinberg's Theorem Revisited; iii. Bulk Loops III: Massless 6D Modes; iv. Embedding into More Fundamental Theories?

(d) Observational Implications: i. Bounds on Extra-Dimensional Radii; ii. Post-BBN Cosmology; iii. Pre-BBN Cosmology; iv. Tests of Gravity; v. Implications for Particle Physics.

\footnotetext{
${ }^{1}$ To appear in the proceedings of the Texas A\&M Workshop on String Cosmology.
} 


\section{DARK ENERGY}

The conventional picture of Big Bang Cosmology was stood on its ear at the close of the 20th Century by observations which established for the first time an observational basis for identifying all of the main contributions to the energy density of the present-day universe.

The cosmic surprise was to find that the universe is dominated by two different kinds of unknown forms of matter [1, 2]. Something like $25 \%$ of the universal energy density turns out to consist of what has come to be known as Dark Matter - a form of nonluminous matter whose existence had been suspected for decades due to its gravitational influence on galaxies and clusters of galaxies. More remarkably, a further $70 \%$ apparently consists of a different kind of unknown substance called Dark Energy [3]. In what may be the ultimate Copernican revolution, ordinary atoms (i.e. baryons) turn out to make up at most only about 5\% of the universe's contents!

The discovery of this Dark Energy has held the theorists' hands to the fire, because it adds a new dimension to a long-standing problem: the cosmological constant problem (for a review of which, see [4]). This problem arises once one tries to embed cosmology into a fundamental theory of small-distance physics, in terms of which the cosmological constant can be interpreted as the vacuum energy density. However given that microscopic theories all tend to predict vacuum energies which are of order the microscopic mass (or length) scales, their predictions are typically 50 orders of magnitude larger than the observed value. The cosmological constant problem then asks why the observed cosmological constant should be so small, and for decades this problem was framed in terms of trying to understand why it should be zero. With the discovery of Dark Energy zero is no longer a good enough answer, and a good theory must also explain why it is nonzero, and yet small.

This first part of the review you are reading summarizes very briefly the evidence for Dark Energy (and Dark Matter), as well as the seemingly insurmountable theoretical problems which its explanation raises. This is followed, in the second part of the review, by a description of what I regard to be a very promising line of approach to solving the cosmological constant problem; one which purports to explain both why it is small and why it is nonzero. The second part describes the progress to date on understanding whether the proposal really works (result: so far, so good), and also briefly describes several of the proposal's many non-cosmological implications, such as for tests of General Relativity on large and small scales and for experiments at high-energy particle accelerators like the Large Hadron Collider (LHC).

\section{Dark Matter and Dark Energy: The Evidence}

As might be expected, widespread acceptance of such a revolutionary picture of the universe has required the concordance of several independent lines of evidence. Although this evidence is still improving, it has led to a remarkably robust picture.

\section{Dark Matter}

The evidence for Dark Matter goes back to the 1930s, and comes from various methods for comparing the amount of matter which gravitates with the amount of matter which is luminous, and so directly visible. Several types of independent comparisons consistently point to there being more than 10 times as much dark material in space than is visible ${ }^{2}$ with the evidence coming from several sources:

- Baryon Abundance Inferred from Nucleosynthesis: The total mass density of ordinary matter (baryons) which can be present within the universe within the Hot Big Bang model can be inferred in two separate ways, independently of any present-day measurements of its gravitational properties. First, the total predicted relative abundance of primordial nuclei within the Hot Big Bang relies on the competition between nuclear reaction rates and the rate with which the universe cools. But the reaction rates depend on the net abundance of baryons in the universe, and the cooling rate depends on the overall expansion rate of the universe, and so - according to General Relativity on its total energy density. The success of the predictions of Big Bang Nucleosynthesis (BBN) therefore fixes the

\footnotetext{
2 This is consistent with the cosmological evidence that Dark Matter is roughly 5 times more abundant than ordinary matter (baryons) because most of the ordinary matter is also dark, and so is not visible.
} 
fraction of the universal energy density which can consist of baryons, and implies that there can only be a few times more baryons out there than what would be inferred by counting those which are luminous, and so directly visible.

- Baryon Abundance Inferred from the Cosmic Microwave Background (CMB): The observation of CMB photons, which come to us from directly from the Big Bang, provide an independent indication of the overall baryon abundance. They do so because these photons were emitted as the universe first became transparent as it cooled enough for electrons and nuclei to form neutral atoms. Any sound waves in the baryon density which were present at this epoch are observable through the small temperature fluctuations which they imply for this CMB radiation, and since the properties of these sound waves depend on the density of baryons at the time, a detailed understanding of the $\mathrm{CMB}$ spectrum allows the total baryon density to be inferred, with a result which is consistent with the results of Big Bang Nucleosynthesis.

- Galaxies: The total mass in a galaxy may be inferred from the observed galactic rotation speeds, measured as a function of the distance from the galactic center. Since the galactic rotation is due to stars and gas clouds orbiting the galactic center, its speed measures the galactic mass distribution in much the same way as Kepler's Laws can be used to infer the masses of planets in our solar system given the orbital properties of their moons. For large galaxies like the Milky Way, the results point to there being several times more matter present than would be obtained by simply counting what is directly visible. Although this matter could consist of baryons without being in conflict with the above bounds on the total baryon abundance, our knowledge of the properties of baryons allows the inference that much of it should be clumped as massive planet-like objects. Direct searches for the microlensing which these objects would produce as they pass in front of more distant stars indicates (weakly) that there are not enough of these kinds of objects to account for the total galactic mass.

- Clusters of Galaxies: The comparison of the amount of visible matter (in galaxies and hot intergalactic gas) in large galaxy clusters with the total mass of the cluster inferred gravitationally points to the existence of much more mass than is visible. Moreover, the amount of mass which must be present is also more than is permitted by the above upper limits on the total baryon density, indicating that the Dark Matter cannot be made of ordinary atoms. The total amount of cluster mass present is inferred in several different ways, such as (i) by finding the depth of the potential well which is required in order to gravitationally bind the hot intergalactic gas, or (ii) by observing the gravitational lensing of more distant galaxies as their light passes by the foreground galaxy cluster.

- Structure Formation: More evidence comes from the assumption that present-day galaxies and galaxy clusters formed due to the gravitational amplification of initially-small primordial density fluctuations. In this case the evidence for Dark Matter arises because of the interplay of two facts: First, the amplitude of initial fluctuations is known to be very small, $\delta \rho / \rho \sim 10^{-5}$, at the time when the universe became transparent because they can be directly inferred from the properties of the observed temperature fluctuations of the cosmic microwave background (CMB) radiation. Second, because small initial fluctuations cannot be amplified by gravity within a radiation-dominated universe, fluctuations cannot begin to be amplified until the epoch where the energy density of non-relativistic matter begins to dominate the more-quickly-falling energy density in radiation. There has not been enough time for these initially-small density fluctuations to form gravitational structure unless there is much more matter present than can be accounted for by baryons. The amount required agrees with the amount inferred from galaxy clusters and from CMB measurements.

We are led to a coherent picture wherein the total amount of Dark Matter is about five times larger than the total amount of baryons which can be present (which is itself several times larger than the amount of visible, luminous matter). Although we do not know what this Dark Matter is, we do know that it is not baryons and that its equation of state (pressure, $p$, as a function of energy density, $\rho$ ) must permit the formation of gravitational structure. This would be true, for instance, if it consisted of an unknown species of particle which does not take part in ordinary nuclear or electromagnetic interactions. If such a particle were sufficiently massive then its pressure would be negligible compared with its energy density: $p \approx 0$, just like for baryons, and it would not hinder structure formation. Better yet, if such a particle were stable and had a mass and interaction strength with other matter which was similar to that of the $Z$ boson (which is not stable), its relic thermal abundance in the Hot Big Bang would naturally be in the observed range [1, 2].

From a theoretical point of view, there seem to be two options for explaining these observations. Since the existence of the Dark Matter is inferred gravitationally, either the laws of gravity are different on extra-galactic scales than those we know and test in the solar system, or there exists a cosmic abundance of a new type of hitherto-undiscovered Dark Matter. 
The current consensus is that it is more likely that Dark Matter is explained by the presence of a new type of particle than by changing gravity on long distances (see however refs. [5, 6, 7] for the best-studied such attempts). This is because we know that it is fairly easy to change the laws of gravity on very short distances, and so long as these changes occur over distances shorter than $\sim 100 \mu \mathrm{m}$ their effects could have escaped detection. But it is very difficult (but not necessarily impossible) to sensibly modify gravity only at longer distances without coming into conflict with observations, or with fundamental principles of relativity or quantum mechanics. Despite many searches for changes to gravity on long distances which can account for the evidence for Dark Matter (and despite reasonable success for galaxy rotation curves alone [5], no completely successful candidate theory has yet been found.

On the other hand, the existence of matter of the type required to be Dark Matter is actually predicted by many current theories of fundamental physics. Such theories often imply the existence of new particles whose interactions and mass are similar to the $Z$ boson, and some of these particles are often stable (such as is true for the lightest supersymmetric particle in many supersymmetric theories, for instance). As mentioned above, this is just what is required to ensure that their present-day relic abundance is what is found for Dark Matter. Although the discovery of such a new stable particle would be a revolutionary development, its properties seem relatively easy to fit into our framework of microscopic theories.

The same seems not to be true for Dark Energy, as we shall now see.

\section{Dark Energy}

Although the evidence for Dark Matter has been accumulating for decades, the big surprise of recent years was the discovery of a second type of dark component to the universal energy density: the Dark Energy. This evidence for the existence of this component comes from two independent lines of argument:

1. Universal Acceleration: Since gravity is attractive, the generic behaviour which is predicted for an expanding universe containing ordinary (and dark) matter $(p \sim 0)$ or radiation $(p=\rho / 3)$ is that its expansion rate should be decelerating. Its expansion decelerates because of the retarding influence of the mutual gravitational attraction of all of the constituent matter and radiation. So it was a surprise when the universal expansion rate was measured at cosmological distances and found to be smaller than it is at present, indicating that the universal expansion is accelerating rather than decelerating. This measurement was made by comparing the observed brightness of various distant supernova, whose intrinsic brightness is believed to be well understood (making them standard candles). Comparing their observed brightness with their known luminosity allows an inference of their distance, and so the universal acceleration is obtained by also performing a measurement of their redshift in order to get the expansion rate at this distance. Since General Relativity implies that an equation of state satisfying $p<-\rho / 3$ would be required in order to make the universal expansion accelerate, neither ordinary matter or Dark Matter cannot be responsible. The matter whose pressure is sufficiently negative to cause this accelerated expansion must be another new form, and is called the Dark Energy.

2. Flatness of the universe: An independent measure of the Dark Energy comes from the observed spectrum of temperature fluctuations in the CMB. This is sensitive to the existence of Dark Energy because the CMB photons have traversed the observable universe before reaching us and so their arrival direction depends on the overall geometry of the universe as a whole. However General Relativity implies that the geometry of the universe also depends on its total energy density, and (taken together with independent measurements of the universal expansion rate) this can be compared with the known (as above) density of ordinary matter and Dark Matter. What is found is that the ordinary matter and Dark Matter abundances fall short by an amount which is consistent with the existence of Dark Energy in the amount required by the Supernova measurements.

From a theoretical perspective, we know that Dark Energy is different from Dark Matter because its equation of state must satisfy $p<-\rho / 3$ (while Dark Matter satisfies $p \sim 0$ ). Interestingly enough, this kind of stress energy can be arranged within relativistic field theories, with the simplest example being given by the spatially-homogeneous motion of a scalar field (or fields), $\phi^{i}(t)[8]$. For such fields $p$ and $\rho$ can be expressed as $p=K-V$ and $\rho=K+V$, where $K=\frac{1}{2} G_{i j}(\phi) \dot{\phi}^{i} \dot{\phi}^{j}$ and $V(\phi)$ are the scalars' kinetic and potential energies. Notice that so long as both $K$ and $V$ are nonnegative, then $\rho \geq 0$ and $-\rho \leq p \leq \rho$. Clearly, the condition $p<-\rho / 3$ in this case requires the scalars' evolution at present to be slow, in the sense that $K<V / 2$. The simplest case in this class is the vacuum energy, for which the scalar field (and so also $p$ and $\rho$ ) is time-independent with $p=-\rho$. In this case the existence of the scalar fields is irrelevant, and the Dark Energy density is purely attributed to the existence of a constant vacuum energy (or 
cosmological constant): $\rho=-p=V$.

So far so good: although the Dark Energy cannot be a gas of relativistic or non-relativistic particles, there are well-motivated kinds of physics whose stress energy can produce a universal acceleration. Furthermore, it is not too difficult to choose the functions $V(\phi)$ and $G_{i j}(\phi)$ in such a way as to account for the known observational features of Dark Energy. Unfortunately, it has nevertheless proven very difficult to embed these models into realistic theories of microscopic physics, for reasons now explained.

\section{Modelling Dark Energy}

The next few sections describe some of the issues which make it difficult to realistically model the universal Dark Energy. The discussion here loosely follows that of refs. [9].

\section{Scales}

What makes Dark Energy more difficult to understand theoretically than is Dark Matter is the size of $K$ and $V$ which are required, together with some generic features of quantized scalar fields. This is because any viable scalar-field model must have two very remarkable properties, which turn out to be quite difficult to arrange. As is explained below, they must have:

- Extremely Small Energy Density, inasmuch as the present value of the scalar potential must equal the observed Dark Energy density: $V \sim \rho \sim\left(3 \times 10^{-3} \mathrm{eV}\right)^{4}$, and;

- Extremely Small Scalar Masses, inasmuch as the scalar's mass must be at most $m_{Q} \sim 10^{-32} \mathrm{eV}$. This second condition does not apply if the Dark Energy is simply a cosmological constant $(K=0)$.

These requirement are very generic properties of explanations of the dark energy in terms of a rolling scalar field. Their necessity may be seen from the scalar field equation of motion within a cosmological context, which may be written:

$$
\ddot{\phi}^{i}+\Gamma_{j k}^{i}(\phi) \dot{\phi}^{j} \dot{\phi}^{k}+3 H \dot{\phi}^{i}+G^{i j}(\phi) \frac{\partial V}{\partial \phi^{j}}=0, \quad \text { and } \quad H^{2}=\frac{1}{3 M_{p}^{2}}\left[\frac{1}{2} G_{i j}(\phi) \dot{\phi}^{i} \dot{\phi}^{j}+V(\phi)+\hat{\rho}\right],
$$

where $M_{p}=(8 \pi G)^{-1 / 2}=10^{18} \mathrm{GeV}$ is the rationalized Planck mass, $G^{i j}$ is the inverse matrix to $G_{i j}, \Gamma_{j k}^{i}(\phi)$ is the Christoffel connection constructed by considering the functions $G_{i j}(\phi)$ to be a metric, and $\hat{\rho}$ is the total energy density due to other degrees of freedom besides $\phi^{i}$. (Recall, however, that it is $V(\phi)$ which is at present dominating in the universal energy density). Physically, the quantity $H=\dot{a} / a$ represents the universal rate of expansion.

It is instructive to quantify what is required of the potential in order to obtain a successful description of the Dark Energy. For this purpose we also restrict our attention to the case of a single scalar field. Since the scalar potential energy must dominate during its motion, it is often a good approximation to suppose that $\phi$ does not move very far from a fixed value, $\phi_{0}$, as it moves. ${ }^{3}$ Using the freedom to redefine fields to choose $\phi_{0}=0$ and $G_{i j}\left(\phi_{0}\right)=\delta_{i j}$ allows us to expand the functions $G_{i j}$ and $V$ as follows:

$$
G_{i j}(\phi)=\delta_{i j}+\cdots, \quad V(\phi)=V_{0}+\frac{m_{\phi}^{2}}{2} \phi^{2}+\frac{\lambda}{4} \phi^{4}+\cdots .
$$

With these choices, we must impose the conditions that the scalar motion be slow enough to be potential-dominated: $M_{p} V^{\prime} \ll V$ and $M_{p}^{2} V^{\prime \prime} \ll V$, and that the value of this potential agree with the observed Dark Energy density: $V_{0} / M_{p}^{2} \sim H_{0}^{2}$, where $H_{0} \sim 10^{-32} \mathrm{eV}$ is the present-day universal expansion rate. As is easily verified, these conditions imply the two constraints quoted above: $V_{0} \sim H_{0}^{2} M_{p}^{2} \sim\left(10^{-3} \mathrm{eV}\right)^{4}$, and $m_{\phi} \ll H_{0} \sim 10^{-32} \mathrm{eV}$.

Provided one is happy to choose these values by hand it is possible to construct phenomenological models of the Dark Energy, and most of the extant models have this spirit. There are two separate difficulties with this point of view,

\footnotetext{
3 This simplifying assumption is not always satisfied, but is also not crucial for the conclusions being drawn here.
} 


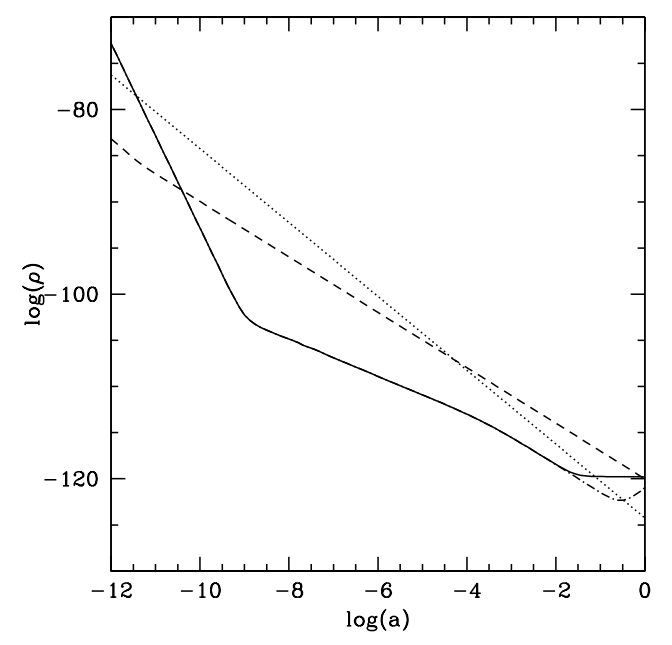

FIGURE 1. The energy density of radiation (dotted), Dark Matter (dashed) and Dark Energy (solid) as a function of universal scale factor, $a$, from the nucleosynthesis epoch until now with the convention that the present epoch is $a=1$.

one of which is cosmological in nature and the other of which is to do with understanding how these numbers arise when we embed the Dark Energy into a realistic theory of fundamental physics. We briefly pause to describe the first problem here, before devoting most of the remainder of this review to the second topic.

\section{Sensitivity to Initial Conditions}

Although the Hot Big Bang model of cosmology is remarkably successful in describing what is seen in observations in terms of a very simple physical picture. It does so, however, only given very particular initial conditions. For instance it must be assumed that the initial universe is extremely homogeneous and spatially very flat. Because evolution within the Hot Big Bang always makes the subsequent universe less homogeneous and less flat than it started, it must have been extremely homogeneous and flat in the past in order to be as homogeneous and flat as it presently is (on large distance scales) at such a ripe old age.

This introduces a worrying sensitivity to a particular set of initial conditions for the success of the Hot Big Bang description. One of the main motivations for considering the possibility that the universe underwent an earlier dramatic inflationary expansion [10] is that it would account for this initial condition for the later universe in a way which can be less sensitive to the details of the initial conditions before this inflationary epoch. This independence of initial conditions can arise because inflationary configurations are often attractor solutions to the system's equations of motion, inasmuch as a broad class of initial conditions all evolve towards the attractor solution.

A drawback of having a time-dependent Dark Energy is that it seems to introduce even more sensitivity to initial conditions, and it is not clear that this new sensitivity can be helped by having an earlier phase of inflationary expansion. To see why this is so consider Figure 1 which gives the evolution from the nucleosynthesis (BBN) epoch until the present of the energy density in radiation, matter and a Dark Energy scalar in the Dark Energy theory of ref. [11], which we can take to be representative for these purposes.

As is clear from this figure, the present epoch is very special in that the energy densities in matter, baryons and Dark Energy are not so different from one another, even though they were extremely different in the cosmological past. (Why this should be true is sometimes called the 'Why Now?' problem.) In the model of ref. [11] the coincidence of these energy densities is ultimately arranged by artfully choosing initial conditions. In particular the initial value of the Dark Energy field is chosen to be close to its present value because it tends not to evolve very far during the epochs after BBN.

One might hope to do better than this, by having the present-day properties of the Dark Energy emerge in an initialcondition-independent way, perhaps as an attractor solution to the relevant field equations. 'Quintessence' models were invented for these purposes, in the hope of explaining why an evolving scalar field could naturally have an energy density which is now so similar to that of other forms of matter [12]. This hope is based on their equations of motion 


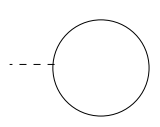

(a)

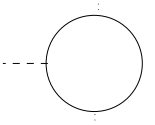

(b)

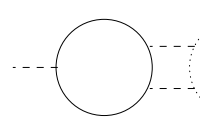

(c)

FIGURE 2. Loop graphs which can generate large quantum contributions to the scalar potential. The dashed line represents a graviton, a dotted line represents the Dark Energy field and a solid line represents a particle of mass $M$.

admitting 'tracking' solutions, within which the scalar energy density closely follows (or tracks) the dominant energy density of the universe as it evolves, making it easier also to understand why the Dark Energy density is presently so close to the Dark Matter and (to a somewhat lesser extent) radiation densities. Since these tracking solutions are often also attractors, they also often describe the late-time evolution of the scalar field regardless of its initial conditions. The existence of tracking, attractor solutions therefore opens the possibility of explaining the present day dark energy in a way which does not rely on the assumption of special initial conditions for the quintessence field in the remote past.

Unfortunately, the devil is in the details and since the other forms of matter do not currently satisfy the Dark Energy equation of state, $p \lesssim-\rho / 3$, neither can the dark energy if it is still in a tracking solution today. So to become the Dark Energy the scalar must eventually leave the tracking solution. Although one might hope that this could also be naturally achieved - perhaps being due to transient behaviour arising from the crossover from radiation to matter domination [13] - so far it has proven difficult to make a completely convincing cosmology along these lines.

It remains an open problem to see whether or not a time-dependent Dark Energy can be described in a way which is insensitive to initial conditions in this way.

\section{Naturalness Issues}

The second - arguably more serious — problem with Dark Energy models only becomes evident when one tries to embed the model into a realistic theory of more fundamental physics. Then one encounters two different kinds of 'fine-tuning' or 'naturalness' problems, which are described in this section and are generic when scalar fields are used in a microscopic context.

\section{The Problem of Quantum Corrections}

In order to see why the small values for $V_{0}$ and $m_{\phi}$ discussed above are problematic when viewed from a more microscopic perspective it is worth comparing the potential of eq. (2) with what is normally found for scalar potentials in microscopic theories.

The theory into which any theory of Dark Energy must be embedded must also contain the Standard Model of the electro-weak and strong interactions (or its close approximation). For more than 20 years, this theory has accounted for (and often predicted) the results of all non-gravitational experiments that have been performed. The only modification which it has required in this time is the addition of the neutrino masses which are required to describe recent discovery of neutrino oscillations. The typical scale in this theory which sets the masses of most of the particles appearing in it is the electro-weak scale, $M \sim 250 \mathrm{GeV}$.

The difficulty with a potential like eq. (2) arises as soon as the Dark Energy field, $\phi$, couples to any Standard Model particles. And these couplings must exist, at least at gravitational strength because Einstein's Equivalence Principle requires all fields to couple to gravity. The problem presented by such couplings is seen once one examines the quantum corrections to $V(\phi)$ to which they give rise. For instance, since the strength of couplings to gravity of a particle of energy $E$ are of order $E / M_{p}$, loop graphs like those of Figures 2 generate the following contributions to $V(\phi)$

$$
\Delta V_{a}(\phi)=\frac{c_{0} M^{4}}{(4 \pi)^{2}}, \quad \Delta V_{b}(\phi)=\frac{c_{2} g^{2} M^{4}}{(4 \pi)^{2} M_{p}^{2}} \phi^{2}, \quad \Delta V_{c}(\phi)=\frac{c_{2}^{\prime} \lambda M^{6}}{(4 \pi)^{6} M_{p}^{4}} \phi^{2},
$$


where $M$ here denotes the mass of the particle which circulated within the loop. Figure $2 \mathrm{~b}$ assumes $\phi$ directly couples to ordinary matter with gravitational coupling strength, $g E / M_{p}$, while Figure 2- assumes only that $\phi$ couples to other fields through the virtual exchange of gravitons.

The factors appearing in these estimates are obtained as follows: There is a standard factor of $(1 / 4 \pi)^{2}$ for each loop (in 4 dimensions); and there is a factor of $1 / M_{p}$ for each graviton vertex (except for the overall tadpole vertex); the factor of $\lambda$ weights the 4-point $\phi$ vertex coming from the classical potential; and the power of $M$ follows on dimensional grounds (in a regularization scheme like dimensional regularization). Provided that $M$ is the largest mass scale in the loop, the dimensionless constants, $c_{n}$, found by explicitly valuating the graph, are generically $\mathscr{O}(1)$.

We see from eq. (3) that even if the coupling between the $\phi$ field and the rest of physics is only gravitational in strength, this coupling generically produces corrections to the quantities $V_{0}$ and $m_{\phi}$ which are bigger than the phenomenologically-chosen values. Since the corrections grow with $M$, they are most sensitive to the most massive virtual particles which can circulate within the loop. For the phenomenologically-acceptable choice $V_{0} \sim\left(10^{-3} \mathrm{eV}\right)^{4}$, Figure 2 $2 \mathrm{a}$ implies the condition $\delta V_{0} \lesssim V_{0}$ is only possible if $M \lesssim\left[(4 \pi)^{2} V_{0}\right]^{1 / 4} \sim 10^{-3} \mathrm{eV}$. Using Figure 2b the condition $\delta m_{\phi} \lesssim m_{\phi}$ similarly requires $M \lesssim\left[4 \pi m_{\phi} M_{p} / g\right]^{1 / 2} \sim 10^{-3} \mathrm{eV} / g^{1 / 2}$. Interestingly, for $g^{1 / 2} \sim \mathscr{O}(1)$ the two scales obtained in this way agree with one another, but they are also clearly many orders of magnitude smaller than the masses of the known elementary particles (apart possibly for neutrinos). Using instead Figure 2: and requiring $\delta m_{\phi} \lesssim m_{\phi}$ gives the weaker condition $M \lesssim 4 \pi\left[m_{\phi} M_{p}^{2} / \lambda^{1 / 2}\right]^{1 / 3} \sim 0.1 \mathrm{GeV} / \lambda^{1 / 6}$. Again (for $\lambda^{1 / 6}=\mathscr{O}(1)$ ) this scale is several orders of magnitude smaller than the masses of most of the known elementary particles.

\section{Two Categories of Fine Tuning}

We see that the quantum corrections to both $m_{\phi}$ and $V_{0}$ are typically much larger than the phenomenologically acceptable values. On the other hand, only the sum of the classical contribution and its quantum contributions is ever measured, since (for example)

$$
V_{0}=V_{0}^{c l}+\Delta V_{0} .
$$

Need one really demand that both $V_{0}^{c l}$ and $\Delta V_{0}$ be separately small? Or might they instead both be large, with one cancelling the other to produce the small observed value?

The existence of such a cancellation is the point of view adopted in some recent discussions of hierarchies [14], because of the discovery of large numbers of supersymmetry-breaking vacua within string theory [15, 16]. The proponents of this attitude notice that different vacua are likely to produce differently-sized local patches of the universe, each having a different local value for quantities like $V_{0}^{c l}$ and $\Delta V_{0}$. If there are sufficiently many such vacua, and if the relative probability of obtaining particular values for $V_{0}^{c l}$ and $\Delta V_{0}$ is distributed fairly smoothly, then there will plausibly be a few vacua for which the cancellation required to obtain a small $V_{0}$ occurs. Then our appearance within such a vacuum is imagined to be explained on anthropic grounds, which argue that things like galaxies can only arise in those regions for which $V_{0}$ is as small as it is, and hence it is only in these vacua that living creatures like ourselves exist [17].

Although this is a logically defensible point of view, it is clearly the explanation of last resort. When thinking about the merits of these arguments I believe it is worth keeping in mind that hierarchies of scale are also known in many other areas of physics, and so we may gain insight from these other examples when thinking about naturalness issues. It is worth rephrasing the issue within the modern picture of the physics of renormalization, which underlies our understanding of what the quantum corrections mean physically [18].

To this end it is more fruitful not to regard the divide between $V(\phi)$ and $\Delta V(\phi)$ as a classical/quantum split. Rather it is more useful to instead think of the 'classical' theory as an effective theory which applies at high energies, and to think of the quantum corrections as being the contributions which are obtained as we 'integrate out' lower-energy degrees of freedom to obtain an effective theory which applies at lower energies. Within such a picture a quantity like $V_{0}^{c l}$ might be the result obtained within the effective theory which applies at energies above the scale $M$ of a particle of interest. The contribution, $\Delta V$, given by eq. (3), comes from integrating out this particle to get the effective theory below the scale $M[19]$. From this perspective it is hard to see why the contributions of high energies should so systematically cancel those of low energies, and so the existence of a small mass scale like $V_{0}^{1 / 4}$ or $m_{\phi}$ raises two separate questions:

- Problem 1: Why is $V_{0}^{c l}$ or $m_{\phi}^{c l}$, so small at the microscopic scales, say $M \gtrsim 10^{3} \mathrm{GeV}$, at which the fundamental theory is couched? 
- Problem 2: Why does the quantity $V_{0}$ or $m_{\phi}$ remain small as all the scales between $M$ and lower energies are integrated out? (That is, why is $\Delta V_{0}$ as small as $V_{0}^{c l}$, or $\Delta m_{\phi}$ as small as $m_{\phi}^{c l}$ ?)

For all of the hierarchies we understand in physics, the smallness of the hierarchy is understood in both of these ways. That is, we understand why the corresponding small parameters are small in both the high-energy theory, and in the low-energy theory obtained by integrating out the intervening physics.

For example, there is a hierarchy of about $10^{5}$ between the radius of a nucleus and the radius of an atom, and in the low-energy theory appropriate to atomic physics (QED) this is understood in terms of the small size of the Bohr wavenumber, $a_{0}^{-1} \sim \alpha m_{e}$, in comparison with the proton mass, $m_{p}$. Here $a_{0} \gg m_{p}^{-1}$ because the electromagnetic coupling, $\alpha=e^{2} / 4 \pi$, is small and because the electron's mass, $m_{e}$ is small compared with the proton's. If we look to higher energies, to the theory (QCD) describing physics inside the nucleus, this hierarchy is understood in terms of the small size of $\alpha$, and because $m_{e}$ is much smaller than the QCD scale, $\Lambda_{Q C D}$. Because both $m_{e}$ and $\alpha$ renormalize logarithmically, they stay small even as the intervening scales are integrated out.

From this point of view we see that it is conservative to also ask that our understanding of the Dark Energy be similarly understood at all scales. In particular we should ask a reasonable theory to explain why quantities like $m_{\phi}$ and $V_{0}$ are small both in the effective theory at the $\mathrm{TeV}$ scale, and why they remain small as all of the known particles having mass $10^{-3} \mathrm{eV}<M<\mathrm{TeV}$ are integrated out. The recent apparent willingness to give up this criterion has largely arisen from the desperation which comes from the great difficulty of doing so in an acceptable way, given that we know so much about such low-energy scales. The main message of this section is to recognize that although anthropic explanations of hierarchies which allow cancellations between high- and low-energy contributions are defensible, they are also radical inasmuch as no previous hierarchies we know need to be understood in this way.

\section{'Technical' Naturalness}

Of the two naturalness questions posed above, it is the Problem 2 which is the more worrying, because it seems to indicate that we are missing something in our description of low energy physics, which we normally think we understand quite well. Hierarchies for which Problem 2, above, is understood are said to be 'technically' natural [20]. A brief summary of the known mechanisms for satisfying technical naturalness for $V_{0}$ and $m_{\phi}$ are now summarized.

The Vacuum Energy, $V_{0}$. The smallness of $V_{0}$ has proven to be the hardest to understand in a technically natural way. Supersymmetric theories provide the only ray of light in what is otherwise a totally dark picture. On the one hand, sufficiently many supersymmetries can explain why the microscopic value, $V_{0}^{c l}$, must be small or vanish (thereby addressing problem 1 , above). On the other hand, one supersymmetry can also partially explain why the process of integrating out lighter particles does not ruin this prediction (problem 2, above), because supersymmetry enforces a cancellation between bosons and fermions in their contributions to $V_{0}$ [21]. Unfortunately, this cancellation is only partial if supersymmetry is broken, leaving a residual nonzero value. If $m_{s b}$ is a measure of the largest mass splittings between bosons and fermions within a supermultiplet, then the residual size of the vacuum energy is typically $\Delta V_{0} \sim m_{s b}^{2} M^{2}$, where $M \gtrsim 1 \mathrm{TeV}$ is the largest mass in the problem. For some theories this leading term (for large $M$ ) vanishes, in which case the residual result can be as small as $\Delta V_{0} \sim m_{s b}^{4}$. Sadly, experiment already implies that $m_{s b}$ must be at least as large as $m_{s b} \sim 10^{2} \mathrm{GeV}$ for observed particles like electrons, so it is has not been clear how to use this fact in a realistic model. We return to how these supersymmetric properties might help produce a technically natural understanding of why $V_{0}$ is so small in the second half of this article, when the proposal of Supersymmetric Large Extra Dimensions (SLED) is discussed in detail.

Cosmologically Light Scalar Fields, $m_{\phi}$. There has been more progress in identifying how a small scalar mass like $m_{\phi} \sim 10^{-32} \mathrm{eV}$ might be technically natural, with three broad classes of ideas having emerged.

1. Pseudo-Goldstone Bosons: The first class of ideas consider the very broad class of scalar models for which $\phi$ is a pseudo-Goldstone boson (PGB) [22]. A PGB is a (would-be) Goldstone boson for an approximate symmetry: i.e. for a symmetry which is both spontaneously broken (at scale $f$ ) and explicitly broken (by terms in the action whose scale is $\mu \ll f$ ). Since any such particle must become massless in the limit $\mu \rightarrow 0$ for which the corresponding symmetry becomes exact, for nonzero $\mu$ its mass is suppressed by powers of $\mu / f$. 
Below the scale $f$ the effective equations of motion for any such a particle has the form of eq. (1), with a kinetic term and a scalar potential of the form

$$
G_{i j}(\phi)=g_{i j}(\phi / f) \quad \text { and } \quad V(\phi)=\mu^{4} U(\phi / f) .
$$

The emergence of the approximate symmetry as $\mu \rightarrow 0$ can be seen here as the appearance of the shift symmetry, $\phi \rightarrow \phi+$ constant, in this limit. ${ }^{4}$ The existence of this symmetry is very important, because it implies that any quantum corrections to $V(\phi)$ must also be proportional to a positive power of $\mu^{4}$, and so must again have the form of eq. (1). This automatically ensures that Problem 2, above, is solved since the quantum corrections to $m_{\phi}$ are naturally as small as is its initial value, $m_{\phi}^{c l}[23]$.

To see how this works in detail, notice that in order of magnitude the scalar potential and its derivatives are given by $V \sim \mu^{4}, \partial V / \partial \phi^{i} \sim \mathscr{O}\left(\mu^{4} / f\right)$ and $V_{i j}=\partial^{2} V / \partial \phi^{i} \partial \phi^{j} \sim \mathscr{O}\left(\mu^{4} / f^{2}\right)$. Expanding $g_{i j}$ and $V$ about $\phi=\phi_{0}$, and using the freedom to redefine fields to choose $G_{i j}\left(\phi_{0}\right)=\delta_{i j}$, implies the scalar mass matrix becomes $m_{i j}^{2}=A_{i}{ }^{k} A_{j}{ }^{l} V_{k l} \sim \mu^{4} / f^{2}$, where $A_{i}{ }^{k} A_{j}{ }^{l} g_{k l}=\delta_{i j}$. We see from this that $m_{\phi} \sim \mathscr{O}\left(\mu^{2} / f\right)$, and a similar argument shows that the quartic scalar self-coupling is $\lambda \sim \mathscr{O}\left(\mu^{4} / f^{4}\right)$. For instance, using this in the above expression for $\Delta V_{c}$ and taking $M \sim f \sim M_{p}$ (more about these choices below) leads to $\Delta m_{\phi}=\mathscr{O}\left(\mu^{2} / 16 \pi^{2} M_{p}\right)$, which is indeed smaller than $m_{\phi}^{c l}=\mathscr{O}\left(\mu^{2} / M_{p}\right)$. (Notice, however, that the shift symmetry does not preclude adding a constant to $V(\phi)$, and so $\Delta V_{0}$ in this case need not be suppressed by powers of $\mu$.)

From a phenomenological perspective the size of the scales $\mu$ and $f$ is fixed by a successful description of Dark Energy, by the following arguments (provided the dimensionless functions $U(x), g_{i j}(x)$ and their derivatives are assumed at present to be $\mathscr{O}(1)$ ). For instance, the value $\mu \sim 10^{-3} \mathrm{eV}$ directly follows because by assumption the total scalar-field potential presently dominates the universal energy density, $\rho \approx V$. In this way we see that $\mu$ controls the present Dark Energy density, $\rho \sim \mu^{4} \sim\left(10^{-3} \mathrm{eV}\right)^{4}$, and so also $H \sim \mu^{2} / M_{p}$. If it is true that $K$ is not too much smaller than $V$ then we also know that $\dot{\phi} \sim \sqrt{K} \sim \sqrt{V} \sim \mu^{2}$. The requirement $f \sim M_{p}$ is determined from the scalar field equation, eq. (1), together with the above slow-roll conditions, since this implies the $\ddot{\phi}^{i}$ and the $\Gamma_{j k}^{i} \dot{\phi}^{j} \dot{\phi}^{k}$ terms should be much smaller than the other two. Except for the case of a pure cosmological constant (for which only the last term is important), we therefore have $H \dot{\phi}^{i} \sim G^{i j} \partial V / \partial \phi^{j}$ and so $\mu^{4} / M_{p} \sim \mu^{4} / f$. From this we learn $f \sim M_{p}$ and hence $m_{\phi} \sim \mu^{2} / f \sim \mu^{2} / M_{p} \sim 10^{-33} \mathrm{eV}$.

The virtue of this kind of model is that it shows how to keep the quantum corrections to the hierarchy between $\mu$ and $f$ - and so also the small size of $m_{\phi} \sim \mu^{2} / f-$ small, and so provide a technically natural solution to Problem 2 for the scalar mass $m_{\phi}$. Unfortunately, these arguments shed no light on why $\mu$ and $f$ should take these values within the high-energy effective theory in the first place - i.e. Problem 1, above. Nor do they provide any understanding of how to solve either Problem 1 or Problem 2 for the vacuum energy, $V_{0}$.

2. Large Kinetic Terms. A related way to solve Problem 2 for the scalar mass is to have $m_{\phi}$ be small due to the largeness of $G_{i j}$ at $\phi=\phi_{0}$ rather than due to the smallness of $V_{i j}$ there [24]. This kind of mechanism relies on the fact that if $G_{i j} \sim \mathscr{O}\left(\varepsilon^{-p}\right) \ll 1$ for $p>0$ and $0<\varepsilon \ll 1$, then the matrices $A_{i}{ }^{j}$ appearing in the mass matrix, $m_{i j}^{2}$, are of order $A_{i}{ }^{j} \sim \mathscr{O}\left(\varepsilon^{p / 2}\right)$. This has the advantage that even if the quantum corrections to $G_{i j}$ are large, they can still be dominated by the still larger contribution of $G_{i j}^{c l}$, and so their size can be technically natural.

3. Small Cutoffs. The third proposal which can make $m_{\phi}$ small enough for Dark Energy obtains if the 4dimensional assumptions which underly the evaluation of the Feynman graphs of Figures 2 break down at sufficiently low energies. This can happen if the largest scale, $M$, for which the effective theory can be 4-dimensional is as low as $M \sim 10^{-3} \mathrm{eV}$, such as can only happen in Large Extra Dimensional models [11, 25].

As we've seen, such a low value for $M$ could resolve the 4-dimensional part of the technical naturalness issue for both $V_{0}$ and $m_{\phi}$. In this approach the most difficult naturalness issues arise in the higher-dimensional effective theory above the scale $M$, a discussion of which is postponed to the more detailed description of Supersymmetric Large Extra Dimensions which follows below.

\footnotetext{
${ }^{4}$ This symmetry also demands a particular form for the kinetic function, $G_{i j}$, whose precise form depends on the symmetry group.
} 


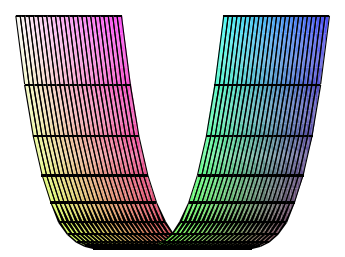

FIGURE 3. A scale-invariant potential with a flat direction along which $V=0$ and scale invariance is broken.

\section{The Problem of 'Self Tuning': Weinberg's No-Go Theorem}

There is another difficulty with obtaining a naturally small Dark Energy density which is related to, but logically distinct from, the above-discussed issues of quantum corrections and technical naturalness. This second problem, first articulated in its general form by Weinberg [4], arises within those proposals which use an underlying scale invariance to try to make the the vacuum energy naturally small. This difficulty is discussed here in some detail because the Supersymmetric Large Extra Dimensional proposal of the next section falls into the class of theories to which Weinberg's objection applies.

Scale invariance frequently arises within Dark Energy proposals because scale invariance is one of two known symmetries - together with supersymmetry - which can force the vacuum energy to vanish. However, whereas supersymmetry can only force the vacuum energy to vanish when it is not spontaneously broken, if scale transformations are a symmetry of the action then the vacuum energy vanishes whether or not the scale invariance is spontaneously broken. It can do so because the vanishing of the vacuum energy is a consequence of conservation of the scale current and so relies only on the invariance of the action. Explicitly, the scale transformation property of the metric, $g_{\mu \nu} \rightarrow \ell^{2} g_{\mu \nu}$, ensures that the conservation condition for a scale invariant theory is $g_{\mu \nu} \delta S_{\text {matter }} / \delta g_{\mu \nu}=0$ once all other fields are evaluated at the solution to their classical equations of motion. This implies quite generally that any scale-invariant scalar potential is automatically minimized at a position in field space for which the potential vanishes: $\partial V /\left.\partial \phi^{i}\right|_{\min }=V_{\min }=0$. This condition ensures in turn that $V$ vanishes at this solution, as required.

It is useful to see why this works in detail in order to appreciate Weinberg's objection. For this purpose it is instructive to consider a collection of scalar fields, $\phi^{i}$, which transform under scale transformations in the canonical way: $\phi^{i} \rightarrow \phi^{i} / \ell$, for which the sole scale-invariant potential is quartic:

$$
V=\frac{\lambda_{i j k l}}{4} \phi^{i} \phi^{j} \phi^{k} \phi^{l}
$$

If we choose the $\lambda_{i j k l}$ so that this potential is strictly non-negative, then it always has a minimum at $\phi_{\min }^{i}=0$ for which $V_{\min }=0$. Unfortunately, the ground state at this point is scale invariant and so the vanishing of $\phi_{\min }^{i}$ also implies all particle masses also typically vanish for this vacuum.

A better situation arises if other vacua exist for which $\phi_{\min }^{i}=v^{i} \neq 0$, in which case scale invariant interactions (like $\mathscr{L}_{\text {yuk }}=g_{i}^{a b} \bar{\psi}_{a} \psi_{b} \phi^{i}$ ) can give particles masses which are proportional to $v^{i}$. Given any one such a vacuum a one-parameter family of vacua may always be generated by performing scale transformations, $\phi_{\min }^{i}=v^{i} / \ell$. Since scale invariance is a symmetry (by assumption) we are guaranteed that all such configurations parameterize a flat direction of the potential $V$, and since the scale-invariant point is included as the limiting case $\ell \rightarrow \infty$ we also know that $V=0$ along this flat direction. This situation is illustrated in Figure 3 and corresponds to the vector $v^{i}$ being a zero eigenvector of the matrix $\lambda_{i j k l} v^{k} v^{l}$, and shows more explicitly why scale invariance ensures a vanishing vacuum energy even if it is spontaneously broken. It is because this offers some hope of understanding why $V_{\min }$ might be much smaller than typical particle masses, $m \sim g_{i} v^{i}$, that scale invariance frequently arises within proposals for understanding why the Dark Energy density can be naturally small. Because of the necessity of having a flat direction, these models also generically predict the existence of a massless (or very light) scalar particle — the dilaton — which can be regarded as being the Goldstone (or pseudo-Goldstone) boson for spontaneously-broken scale symmetry. 


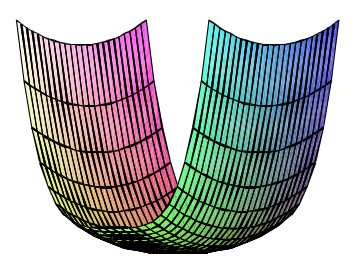

FIGURE 4. A scale-invariant potential along which the flat direction is lifted, with scale invariance not broken at the minimum.

We can now also see the weakness of this proposal. Although spontaneous scale breaking is easily arranged, because it involves a flat direction we must check that this flat direction is not lifted by quantum corrections. For internal symmetries the flat direction which underlies spontaneous symmetry breaking is normally guaranteed by the symmetry itself but this is not so for scale invariance, as can be easily seen for the scalar example under consideration. Even if we assume there to be no quantum anomalies to the scale symmetry, the scale invariance of the quantum action dictates that quantum corrections to $V$ must have the form of eq. [6], but with $\lambda_{i j k l}$ replaced by a quantum-corrected coupling, $\lambda_{i j k l}^{\text {eff }}$. But scale invariance by itself does not impose additional conditions on these couplings and so in particular cannot ensure the existence of the flat direction. Generically even scale-invariant quantum corrections will lift this flat direction, leading to the situation of Figure 4 and so although they do not make the vacuum energy nonzero, they do so by ensuring that the only vacuum is the uninteresting scale-invariant point: $\phi_{\min }^{i}=0$.

It is clear that this objection is quite generic, and although it was encountered in numerous models in the 1980's [26] Weinberg most effectively emphasized how general it is. At face value, scale invariance must climb two hurdles in order to help with the cosmological constant problem: it must survive quantization (i.e. scale anomalies must cancel); and a separate mechanism must be found which keeps the required flat direction flat. Otherwise the theory predicts a vanishing vacuum energy, plus vanishing particle masses of all kinds. Phrased this way, we can see that supersymmetry may be able to help since (if it is unbroken) it can be good at producing flat directions. Unfortunately, the typical curvature of a flat direction which is generated by quantum corrections in a supersymmetric theory are also of order the supersymmetry-breaking scale, $m_{s b}$, which appears to be too large given what is known about elementary particle masses.

We shall see in the next sections how this last conclusion can be evaded within special sorts of brane-world models, and indeed the ability to thread the No-Go result is one of the principal motivations for this proposal. As is discussed in more detail below, the main reason these brane-world models can do so is because in these models the scale of supersymmetry breaking for the dilaton can be made to be much smaller than the supersymmetry breaking scale for ordinary particle multiplets. As a result it is possible for the supersymmetry breaking scale in the dilaton sector to be as small as the cosmological constant scale, $10^{-3} \mathrm{eV}$, even though there are no supersymmetric partners for the observed particles which are light enough to be visible in particle accelerators.

\section{SUPERSYMMETRIC LARGE EXTRA DIMENSIONS}

The remainder of this article describes a very promising proposal [27, 28, 29, 30, 31] - Supersymmetric Large Extra Dimensions, or SLED for short - for solving the cosmological constant problem and understanding the very small size of the Dark Energy density, $\rho$. This proposal turns out to predict that the Dark Energy is at present dynamical, and also naturally addresses the issue of why the rolling scalar fields can be so incredibly light.

As we shall see, the SLED proposal also has a host of other robust phenomenological implications besides those it has for cosmology. These include:

- Deviations from the inverse square law for gravity, which more precise estimates show should arise for distances of order $r / 2 \pi \sim 1 \mu \mathrm{m}$; 
- A particular scalar-tensor theory of gravity at large distances, with the scalar being the moduli (like the volume) which describe the two large extra dimensions. This is the same scalar whose time-dependence now describes the Dark Energy.

- Distinctive missing-energy signals in collider experiments at the LHC [32, 33] due to the emission of particles into the extra dimensions.

- Potential astrophysical signals (and bounds) due to the possibility of having too much energy loss into the extra dimensions by stars and supernovae [2, 34, 35, 32].

If the SLED proposal is correct, it will be spectacularly so since it requires this entire suite of observational implications to be found. Indeed, it is this unprecedented connection between observables in cosmology and particle physics which is driven by its addressing the fundamental naturalness issues described in previous sections - that sets the SLED proposal apart from other descriptions of Dark Energy.

\section{The Proposal}

The two features which define the SLED proposal are large (i.e. $r \sim 10 \mu \mathrm{m}$ ) extra dimensions, arising within a supersymmetric theory.

Large Extra Dimensions. The SLED proposal [27, 29] — like the LED proposal before it [36, 37] — posits that at present there are two extra dimensions whose circumference, $r$, is of order $r \sim 10 \mu \mathrm{m} \sim\left(10^{-2} \mathrm{eV}\right)^{-1}$. This is only possible within the framework of the brane-world scenario according to which all of the observed non-gravitational particles are trapped on surfaces (the 'branes') within spacetime, with only the gravitational degrees of freedom being free to explore the spacetime full extent (the 'bulk') away from these surfaces. The motivation for considering such a possibility is that it can emerge relatively naturally from some string theories, such as if the Standard-Model-like spectrum of massless particles comes from open strings which are localized near very particular kinds of surfaces (' $D$ branes') within spacetime [38]. Although the branes encountered within string theory tend to have very specific kinds of properties a more phenomenological approach is adopted here, wherein we try to identify which brane properties are required to solve the cosmological constant problem. If any such brane properties can be identified, then the ultimate goal is to try to see whether branes having these properties can be found within a more fundamental theory (like string theory).

A second motivation for working within this kind of brane-world proposal is the success it has had in recasting low-energy naturalness issues by identifying unnecessarily restrictive hidden assumptions which physicists had made about the nature of the low-energy world. Branes provide the 'existence proof' that reasonable theories of shortdistance physics can produce much more complicated dynamics at low energies than had been hitherto entertained. The SLED proposal focuses a particular hidden assumption which might be behind the cosmological constant problem: the assumption that physics is four dimensional at energy scales above the cosmological-constant scale, $v \sim 10^{-3} \mathrm{eV}$. After all, this assumption is crucial to the problematic statement that a particle of mass $m$ contributes an amount $\Delta V_{0} \sim m^{4}$. If the world were to involve extra dimensions as large as $r \sim 1 / v \sim 0.1 \mathrm{~mm}$, then any calculation of the contribution of a particle having mass $m>v$ (such as the electron) must be done within a higher-dimensional theory for which the predictions could differ.

The possibility that extra dimensions can be this large without being in conflict with experimental observations is based on the earlier non-supersymmetric large-extra-dimensions (LED) proposal [36], whose authors first realized that it was possible. The possibility only arises within a brane-world framework because in this case only gravitational interactions can probe the existence of the extra dimensions. In an interesting numerological coincidence, it happens that the current limit on the size of such extra dimensions, based on the best present measurements of the gravitational inverse-square law [39], is $r / 2 \pi \lesssim 0.1 \mathrm{~mm}$ (which is of order $1 / v$ ). ${ }^{5}$

In a second extraordinary numerical coincidence, this same radius also accounts for the small ratio, $M / M_{p}$, between the weak scale, $M$, and the Planck scale, $M_{p}$ - i.e. the electro-weak hierarchy problem. This is because Newton's constant, $8 \pi G=1 / M_{p}^{2}$, is related to $r$ by $^{6} M_{p}=M_{g}^{1+n / 2} r^{n / 2}$, where $M_{g}$ is the scale of gravity in the extra dimensions

\footnotetext{
5 Notice that we use the Jordan frame, for which $M_{p}$ is $r$-dependent but the electroweak scale, $M$, is not. Furthermore, our conventions are such that $r^{n}$ is the 2D volume, and so for torii the mass this implies for a Kaluza-Klein (KK) particle is $M_{\mathrm{KK}} \sim 2 \pi / r$.

6 The supersymmetry breaking scale would be larger - for fixed $M_{p}$ - if the extra dimensions should be warped [28].
} 
and $n$ is the number of extra dimensions whose size is much larger than $1 / M_{g}$. In particular, for $n=2$ this requires the extra dimensions to have $r \sim 1 / v$ if the scale of gravitational physics in the extra dimensions is of order the electro-weak scale: $M_{g}=\left(M_{p} / r\right)^{1 / 2} \sim 10 \mathrm{TeV} \sim M$.

Supersymmetry. SLED also supposes these large extra dimensions to arise within a supersymmetric field theory, such as might be expected to be obtained in the low-energy limit of string theory. In such a picture supersymmetry must be badly broken on our brane, since we know that there are no super-partners for the observed particles having masses which are much smaller than $M_{g}$. Given this scale for supersymmetry breaking on the brane there is also a trickle-down of supersymmetry breaking to the 'bulk' between the branes, whose size is set by the bulk's KaluzaKlein scale, $m_{s b} \sim M_{\mathrm{KK}}$, and so which for unwarped geometries can be as low as $m_{s b} \sim 1 / r \sim 10^{-2} \mathrm{eV}$ [32, 27]. Much of the success of the SLED proposal relies crucially on the ability to maintain this hierarchy between the scales of supersymmetry breaking on the brane and in the bulk [40].

\section{The Action}

In more detail, the action for any SLED variant has the form

$$
S_{\mathrm{tot}}=S_{S G}+\sum_{b} S_{b}
$$

where $S_{S G}$ describes the action of one of the 6-dimensional supergravities, and $S_{b}$ contains the physics on one of possibly many 3-branes, on of which we find ourselves situated.

The Bulk. The physics of the bulk, $S_{S G}$, is described by one of the varieties of $6 \mathrm{D}$ supergravity. This supergravity can either be chiral [41] or non-chiral [42], and can be gauged or ungauged (in the sense that a particular $R$-symmetry which does not commute with supersymmetry can arise as a gauge or a global symmetry). The field content of the 6D gravitational supermultiplet typically consists of a scalar dilaton $(\phi)$, two symplectic-Weyl spin-1/2 dilatini $\left(\chi^{r}, r=1,2\right)$, a 2 -form gauge potential $\left(B_{M N}\right)$, two symplectic-Weyl gravitini $\left(\psi_{M}^{r}\right)$ and a metric $\left(g_{M N}\right){ }^{7}$ Chiral and non-chiral models differ in whether or not the two bulk dilatini, $\chi$, (or the two bulk gravitini, $\psi_{M}$ ) share the same 6D chirality: $\Gamma_{7} \chi^{r}= \pm \chi^{r}$.

Additional matter multiplets can also be present, including hyper-multiplets ( 2 real scalars, $\Phi^{i}$, and a symplecticWeyl spin-1/2 particle), gauge multiplets (a gauge boson, $A_{M}^{a}$, plus 2 symplectic-Weyl spin-1/2 fermions), tensor multiplets, massive KK multiplets, and so on. For the present purposes we take the gauged, chiral 6D theory coupled to gauge multiplets as being representative, for which the bosonic action is

$$
\frac{\mathscr{L}_{S G}}{e_{6}}=-\frac{1}{2} R-\frac{1}{2} \partial_{M} \phi \partial^{M} \phi-\frac{1}{2} G_{i j}(\Phi) \partial_{M} \Phi^{i} \partial^{M} \Phi^{j}-\frac{e^{-2 \phi}}{12} G_{M N P} G^{M N P}-\frac{e^{-\phi}}{4} F_{M N}^{a} F_{a}^{M N}-v(\Phi) e^{\phi},
$$

in units for which $M_{g}=1$ : i.e. $M_{g}^{-4}=8 \pi G_{6}=1$. As usual, in this expression $e_{k}=\sqrt{-\operatorname{det} g_{M N}}$ in $k$ spacetime dimensions. The hypermultiplet potential, $v(\Phi)$, has a minimum at $\Phi^{i}=0$ for which $v(0)=2 g^{2}$, where $g$ denotes the gauge coupling of the specific $R$ symmetry for which none of the bosons listed above transforms (although their superpartners do).

The Branes. The cosmological-constant mechanism described below does not depend on many of the details of $S_{b}$. For phenomenological purposes it should be emphasized that because our brane breaks supersymmetry, this symmetry is only nonlinearly realized [43] and in particular does not imply the existence of superpartners for ordinary particles in the usual sense. Rather, a supersymmetry transformation of a light brane state (like the electron) instead produces a massless Goldstone fermion, which is eaten by the massless Kaluza-Klein mode of the bulk gravitino to give this particle its mass. Since nonlinearly-realized gauge symmetries (like supergravity) are indistinguishable at low energies from explicitly broken ones [44], the result is an effective theory of brane-bound states at $\mathrm{TeV}$ energies which appears to explicitly break supersymmetry [45].

${ }^{7}$ Strictly speaking, this multiplet is reducible, since a smaller gravity multiplet can be built using only the metric, gravitino and the self-dual piece of $B_{M N}$. 
In the minimal SLED version (MSLED) one makes the particularly simple choice that the action on our brane is given (apart from terms which couple brane and bulk fields) by precisely the Standard Model [30], but although this is particularly predictive in its implications for collider experiments it is not a required choice.

For reasons which will become clear in what follows, it will turn out in many cases that the bulk dilaton, $\phi$, is related to the extra-dimensional volume by $e^{\phi} \sim\left(M_{g} r\right)^{-2} \ll 1$, and so we must also suppose that gauge couplings amongst the physical fields on our brane do not vanish in the limit that the bulk dilaton runs away to asymptopia: $e^{\phi} \rightarrow 0$. That is, if the physical coupling of brane-bound gauge fields is denoted $g_{b}$, then we must demand $g_{b}=g_{b}^{0}+g_{b}^{1} e^{\phi}+\cdots$, with $g_{b}^{0} \neq 0$. As we shall see below, arranging for this to be true in the Einstein frame may be difficult to accomplish within string theory, depending on the relation between the $6 \mathrm{D}$ dilaton and the underlying string coupling.

\section{Classical Relaxation of the 4D Cosmological Constant}

Within the above framework gravitational physics is effectively 6-dimensional for any energies above the scale, $1 / r \sim 10^{-2} \mathrm{eV}$, and so the cosmological constant problem must be posed within this new context. In order to see how the cosmological constant problem is phrased in 6 dimensions, one must integrate out the degrees of freedom between the scales $M_{g} \sim 10 \mathrm{TeV}$ and $1 / r \sim 10^{-2} \mathrm{eV}$. We seek the cosmological constant within the effective 4D theory obtained after performing this integration, which describes gravitational physics (like present-day cosmology) on scales much larger than $r$. Imagine, therefore, performing the integration over modes having energies $1 / r<E<M_{g}$ in the following three steps [27]:

1. First, integrate out (exactly) all of the degrees of freedom on the branes, to obtain the low-energy brane dependence on the massless 4D graviton mode. In so doing we obtain (among other things) a large effective brane tension, $T \sim M_{g}^{4}$ for each of the 3-branes which might be present, which includes the vacuum energies of all of the presently-observed elementary particles.

2. Next, perform the classical part of the integration over the bulk degrees of freedom. This amounts to solving the classical supergravity equations to determine how the extra dimensions curve in response to the brane sources which are scattered throughout the extra dimensions. It will be argued that it is this classical response which cancels the contributions from the branes obtained in Step 1, above.

3. Finally, perform the quantum part of the integration over the bulk degrees of freedom. Given the cancellation of the previous two contributions, it is this contribution which is responsible for the fact that the present-day Dark Energy density is nonzero. It is argued below that in some circumstances this quantum contribution is of order $m_{s b}^{4}$, where $m_{s b} \sim M_{g}^{2} / M_{p} \sim 10^{-2} \mathrm{eV}$ is the supersymmetry-breaking scale in the bulk. The small size of the $4 \mathrm{D}$ vacuum energy is in this way attributed to the very small size with which supersymmetry breaks in the bulk relative to the scale with which it breaks on the branes.

In a 4D world, the only contribution we would have is that of Step 1, above, and the problem is that this is much too large. But in a 6D world, because all of the observed particles are localized on our brane their vacuum energy should be thought of as a localized energy source in the extra dimensions, to which the bulk geometry must respond. The next section argues that the classical part of this bulk response (Step 2, above) is of the same order as the direct contribution of Step 1, and precisely cancels it in a way which does not depend on the details of the supergravity involved or of the precise extra-dimensional geometry which lies between the various branes. In this way it provides a 6 dimensional realization of self-tuning, whereby the effective $4 \mathrm{D}$ cosmological constant is automatically adjusted to zero by the classical response of the $2 \mathrm{D}$ bulk to the brane sources. The size of quantum bulk contributions are discussed in more detail below, after first pausing to discuss the nature of this classical self-tuning.

\section{D Self-Tuning: A Cartoon}

Step 1 consists of exactly integrating over all brane fields having masses larger than $1 / r$, and this produces a variety of local interactions in the effective theory for energies $E \lesssim 1 / r$ on the brane. Since our interest is in the dependence of the effective theory on the 4D metric, and we assume a large volume for the extra 2 dimensions $-M_{g} r \gg 1-$ we may expand these effective interactions in powers of the curvature:

$$
\delta \mathscr{L}_{b}=-\sqrt{-g}\left[\delta_{b} T_{b}+\frac{1}{2} \delta_{b} \mu_{b}^{2} R+\cdots\right],
$$


where on dimensional grounds we expect $\delta_{b} T \sim M_{g}^{4}, \delta_{b} \mu_{b}^{2} \sim M_{g}^{2}$ etc. ${ }^{8}$

Step 2 consists of the classical part of the bulk integration, and so is equivalent to substituting into the classical action the bulk field configurations which are found by solving the classical field equations using the above effective brane action as a source. It happens that for many situations this classical response of the extra dimensions can be computed explicitly within the approximation that the branes are regarded as delta-function tension sources. Because 3 -branes in 6 dimensions have co-dimension 2, the discussion resembles the better-known analysis of the gravitational field of a cosmic string in 4 dimensions [46].

For co-dimension 2 the extra-dimensional curvature tensor typically acquires a delta-function singularity at the position of the branes, corresponding to a singularity at the brane position. ${ }^{9}$ Einstein's equations require that the singular contribution to the two-dimensional curvature given by

$$
\sqrt{g_{2}} R_{2}=-2 \sum_{b} T_{b} \delta^{2}\left(y-y_{b}\right)+\text { (smooth contributions), }
$$

Here $y_{b}$ denotes the position of the ' $i$ ' th brane in the transverse 2 dimensions, and the 'smooth contributions' are all of those which do not involve a delta-function at the brane positions.

The effective 4D cosmological constant after performing Steps 1 and 2 above is obtained by plugging the above expression into the classical bulk action, eq. (8). The effective 4D cosmological constant obtained at this order is then

$$
\rho_{\mathrm{cl}}=\sum_{b} T_{b}+\int_{M} d^{2} y e_{2}\left[\frac{1}{2} R_{2}+\ldots\right]=0,
$$

where the sum on ' $b$ ' is over the various branes in the two extra dimensions and '...' denotes all of the other terms besides the Einstein-Hilbert term in the supersymmetric bulk action. The final equality here has two parts. First, the sum over brane tensions, $T_{b}$, precisely cancels the contribution of the singular part of the curvature, eq. (10), to which they give rise [47]. Second, a similar cancellation also occurs amongst the various 'smooth' contributions in $\rho_{\mathrm{cl}}$ once these are evaluated for all of the bulk fields using the classical field equations [27]. Interestingly, this cancellation does not depend on the details of the bulk geometry, or on the number of branes, since it relies only on a classical scale invariance which all 6D supergravity actions enjoy [28]. Best of all, this cancellation does not depend at all on the value of the brane tension, $T_{b}$, and so applies equally well even if these tensions are large and include all of the quantum effects due to virtual particles localized on the branes.

We are left with the contribution of quantum effects in the bulk (Step 3), to which we return in more detail in subsequent sections. These must ruin the brane-bulk cancellation because the scale invariance of the classical supergravity equations is not a bona-fide quantum symmetry. However the bulk sector of the theory is also one which is almost supersymmetric, since the bulk supersymmetry-breaking scale is very small: $m_{s b} \sim 1 / r \sim 10^{-2} \mathrm{eV}$. As a result we might expect standard supersymmetric cancellations to suppress the quantum part of the result by powers of $m_{s b}^{2}$, and if the leading term should be of order $m_{s b}^{4}$ this would be the right size to account for the observed Dark Energy density. As is shown in later sections, under certain circumstances this is indeed what happens for some 6D supergravities.

Before evaluating the quantum contributions of Step 3, we first take a more detailed look at the classical 6D selftuning mechanism which was just described.

\section{D Self-Tuning I: General Solutions}

The very name 'self-tuning' carries a whiff of swindle, partly because previous self-tuning proposals [48] were later found really to be cosmetic fixes [49], which merely hid the tuning of the cosmological constant to zero. It is natural to suspect a similar swindle is occurring here, and so the present section describes some of the recent steps which have been taken to rule out hidden fine-tunings within the classical part (Steps 1 and 2) of the argument given above. These steps were made possible by the explicit construction of a very general class of solutions to the chiral, gauged supergravity in 6 dimensions [50]. These solutions are now summarized, with a view to seeing whether or parameters need be specially adjusted in order to keep the effective 4D cosmological constant zero.

\footnotetext{
${ }^{8}$ The subscript ' $b$ ' on $\delta_{b}$ here distinguishes these from similar, but independent, contributions to the localized brane action which arise from integrations over short-wavelength bulk modes (i.e. when performing Steps 2 and 3).

9 The generality of this form of singularity is a question which is examined more closely in later sections, following the discussion of ref. [31].
} 
Explicit Solutions. The simplest explicit solutions to 6D chiral, gauged supergravity in the presence of source branes is the 'rugby-ball' solution of ref. [27], ${ }^{10}$ which consists of product-space metric

$$
\mathrm{d} s^{2}=\eta_{\mu v}(x) \mathrm{d} x^{\mu} \mathrm{d} x^{v}+\mathrm{d} s_{2}^{2},
$$

where $\eta_{\mu v}$ is the Minkowski-space metric and $\mathrm{d} s_{2}^{2}=r^{2}\left(\mathrm{~d} \theta^{2}+\sin ^{2} \theta \mathrm{d} \varphi^{2}\right)$ is the line element on a 2-sphere of radius $a$. This solves the supergravity equations obtained from eq. [8], with constant dilaton, $\phi_{0}$ and a Maxwell field strength, $F_{m n}=f \varepsilon_{m n}$, which is proportional to the 2D volume form. For these choices the field equations imply the parameters $\phi_{0}, f$ and $r$ must satisfy $g^{2} e^{\phi_{0}} \propto g f \propto 1 / r^{2}$. The parameter $r$ is undetermined by the field equations, and parameterizes the flat direction which is guaranteed to exist at the classical level due to the classical scaling symmetry of the supergravity field equations.

Branes are incorporated into this solution by removing a wedge from the 2-sphere whose edges are lines of longitude and identifying these edges once the wedge is removed. This removal and identification introduces a conical singularity to the North and South poles of the 2-sphere, which represent the gravitational back-reaction of the branes onto the bulk geometry. Notice in particular that because the defect angles at the north and south poles are the same size for the rugby-ball solution, the tension of each of the branes situated at these points must be equal to one another. We wish to ascertain whether or not these kinds of constraints constitute the hidden fine-tunings which are responsible for keeping the 4 dimensions flat. More general solutions to the 6D supergravity field equations - for both the chiral and non-chiral theories - have also been found [28], as well as new kinds of solutions to the non-supersymmetric theories [51], for which the large 4 dimensions are warped and the small internal 2 dimensions are squashed.

The best test of the above self-tuning argument at present available is based on a class of solutions to 6D chiral, gauged supergravity obtained in ref. [50] by Gibbons, Guvens and Pope (henceforth GGP). What makes these solutions so useful as a test of self-tuning is that these authors derive the most general solution to these field equations subject to two assumptions: (i) maximal symmetry in 4 dimensions (i.e. de Sitter, Minkowski or anti-de Sitter space), and (ii) axial symmetry in the internal 2 dimensions. That is, they find the most general solutions whose metric has the form

$$
\mathrm{d} s^{2}=W^{2}(\theta) g_{\mu v}(x) \mathrm{d} x^{\mu} \mathrm{d} x^{v}+\mathrm{d} \theta^{2}+a^{2}(\theta) \mathrm{d} \varphi^{2},
$$

and for which $\phi=\phi(\theta)$ and $A_{\phi}=A_{\phi}(\theta)$. Here the intrinsic 4D metric, $g_{\mu \nu}$, satisfies $R_{\mu \nu \lambda \rho}=c\left(g_{\mu \lambda} g_{v \rho}-g_{\mu \rho} g_{v \lambda}\right)$, for some constant $c$. The assumed axial symmetry corresponds to shifts of the coordinate $\varphi$, and the metric can have singularities at up to two positions, $\theta=\theta_{ \pm}$, within the internal 2 dimensions corresponding to the positions of source branes. GGP find that there is a five-parameter family of solutions to the supergravity equations subject to these symmetry conditions. What is most remarkable about these solutions is that every single one of them has a flat intrinsic $4 D$ geometry (i.e. $c=0$ ), even though none of them is supersymmetric (except for the single spherical solution with no wedge removed, mentioned above), as is consistent with the expectation that the effective 4D cosmological constant vanishes.

Given a class of general solutions it becomes possible to begin to address the question of whether hidden finetunings are responsible for this $4 \mathrm{D}$ flatness. In particular, are the brane tensions and couplings being carefully adjusted by an unseen sleight of hand? A partial answer to this question is now possible, and although at first sight such an adjustment appears to take place, it is now explained why these appearances may be deceiving [31].

In order to do so it is useful to keep track of the physical meaning of the parameters on which the general GGP solutions depend. There are 5 such parameters, but one of these simply parameterizes the flat direction whose existence is guaranteed by the classical scale invariance of the supergravity equations. A second parameter corresponds to another classical scaling property, under which a redefinition of the fields may be used to rescale the gauge coupling $g$ to any positive fixed value. The three remaining parameters are broadly related to the three physical quantities which characterize these geometries: the tensions, $T_{ \pm}$, of the two branes which source the bulk geometry; and the overall magnetic flux of the background magnetic field which (marginally) stabilize it.

We now ask what may be said about the natural of self-tuning given the properties of these general solutions. What is at first sight disturbing is that for the generic GGP solutions these parameters are always subject to a topological constraint, and those solutions for which the metric singularities are purely conical are subject in addition to a second, nontopological constraint [28, 31]. Since either (or both) of these superficially might be thought to be the hidden self-tuning, we next discuss each of them in turn.

${ }^{10}$ Similar solutions within non-supersymmetric theories are considered in ref. [53]. 
Topological Constraints. The only constraints which hold quite generally for the GGP solutions are those which have their origins in topology. In fact, there are two topological conditions which these solutions all share: one which expresses that the internal 2D geometry is topologically a sphere; and one which expresses the quantization (and conservation) of magnetic monopole flux [27, 52]. ${ }^{11}$ Since the first of these turns out to hold for all values of the parameters describing the classical solution [50], it is of less interest as a potential source of fine-tuning. The quantization of monopole number potentially has more to say in this regard, since it directly imposes a relation between the brane tensions, the gauge couplings and one of the 5 parameters characterizing the background solutions.

The resulting topological constraint can be written in the following way [31]:

$$
\frac{g^{2} e^{-\phi_{0} / 2}}{2}\left(\frac{T_{+}-T_{-}}{4 \pi}\right)=N^{2}\left(\frac{g^{2}}{\tilde{g}^{2}}\right),
$$

where $N$ is the integer which labels the monopole number. Here $T_{ \pm}$are the two brane tensions, $g$ is the gauge coupling which appears explicitly in the 6D supergravity action, eq. (8), $\tilde{g}$ is the gauge coupling for the background magnetic field and $\phi_{0}$ is an additive constant in the dilaton configuration (and so is one of the parameters describing the solution). The limit of equal tensions, $T_{+} \rightarrow T_{-}$, of this expression involves a subtlety, because one must also take $g^{2} e^{-\phi_{0} / 2} \rightarrow \infty$ — with the product $\left(T_{+}-T_{-}\right) g^{2} e^{-\phi_{0} / 2}$ fixed - in order to keep the explicit solution well-defined. If both limits are taken then the rugby-ball solution discussed above is obtained, with $T_{ \pm}=4 \pi(1-k)$ for some $k$. In this case the above topological constraint degenerates to $k^{2}=N^{2}\left(g^{2} / \tilde{g}^{2}\right)$.

It is tempting to conclude that this monopole-number constraint is the hidden fine-tuning we seek, since it explicitly requires the brane tensions appearing in the solution to be adjusted relative to one another. But first impressions deceive, and this initial conclusion does not stand up to closer scrutiny, as is now argued (following refs. [28, 29]). Recall in this regard that the crucial issue for fine-tuning is whether or not the constraint is stable against renormalization. That is, if eq. (14) is imposed amongst the renormalized quantities at the TeV scale, does it automatically remain imposed as successive scales are integrated out down to the scales below $1 \mathrm{eV}$ ? If so, then the constraint is technically natural, in the sense described above, and so is not fine-tuned this (the most serious) notion of fine tuning. But topological constraints are always natural in this sense, because the integrating out of successive scales of physics is a continuous process, and precisely because topological constraints involve quantization of quantities in terms of integers, they remain unchanged by any such continuous process. Although the functional form of the topological constraint in terms of the renormalized parameters of a theory may change with scale, the validity of the topological constraint itself is guaranteed, by continuity, to be stable under renormalization.

So what do these topological constraints mean? Their meaning is probably most clear if the branes are allowed to couple directly to the background electromagnetic flux, through a term in the brane action of the form [28]

$$
\Delta S_{b}=q_{b} \int^{\star} F,
$$

where ${ }^{\star}$ denotes the 6D Hodge dual of the 2-form field strength. (Such a coupling is not turned on for the GGP solutions under discussion.) In the presence of such couplings the topological constraint of eq. (14) instead states that the difference $q_{+}-q_{-}$is proportional to $\frac{1}{2} g^{2} e^{-\phi_{0} / 2}\left(T_{+}-T_{-}\right) / 4 \pi-N^{2}\left(g^{2} / \tilde{g}^{2}\right)$, which reduces to eq. (14) in the limit where $q_{+}=q_{-}$. Seen in this way, the topological constraint restricts the kinds of magnetic couplings which are possible on topological grounds, in much the same way that topology only allows electrostatic potentials to be constructed for a collections of charges within a compact space, if the sum of all the charges vanishes. That is, it is the integrability condition for the existence of a solution to the field equations subject to the given boundary conditions.

Conical Singularities. Although only the topological constraints holds quite generally for all of the GGP solutions, there is another issue which may bear on whether or not the solutions involve a hidden fine tuning [31]. This additional issue concerns the kinds of metric singularities to which physically reasonable 3-branes can give rise within 6D supergravity. In order to understand the issue, recall the discussion of the gravitational field of a cosmic string in 4 dimensions (using pure Einstein gravity) [46]. In this situation there are two solutions to the Einstein equations which have the symmetries required to be the field of a cosmic string. One of these is flat space, and the other is a curved space whose geometry resembles the shape of the mouth of a trumpet [54]. Of these, the flat solution exhibits a conical singularity at the string position (in the limit of an infinitely thin string), while the trumpet-shaped solution

\footnotetext{
${ }^{11}$ See also [53] for a similar discussion in the non-supersymmetric case.
} 
has a real curvature singularity there. In principle, which solution applies in any physical situation can be determined by resolving the internal structure of the string, in order to smooth out the metric at the string's center. This can be done explicitly, for example, in terms of gauge and scalar fields if the string is a weakly-gravitating Nielsen-Oleson string within a gauge theory. In this case it can be proven that only the flat solution (with conical singularity) can be smoothly joined to the smooth geometry of the string's interior [55].

If it is true that only conical singularities can also be produced by physically reasonable 3-branes in 6 dimensions, then a hidden fine-tuning might underly the vanishing $4 \mathrm{D}$ cosmological constant within $6 \mathrm{D}$ supergravity. This is because only a subset of the general GGP solutions involve purely conical singularities, ${ }^{12}$ defined by the family of GGP solutions whose tensions satisfy the condition [28, 31]:

$$
\left(1-\frac{T_{+}}{4 \pi}\right)\left(1-\frac{T_{-}}{4 \pi}\right)=\frac{g^{2} e^{-\phi_{0} / 2}}{2}\left(\frac{T_{+}-T_{-}}{4 \pi}\right)=N^{2}\left(\frac{g^{2}}{\tilde{g}^{2}}\right) .
$$

Here the last equality follows from using the topological constraint, eq. 14. (Recall that the limit of equal tensions the rugby ball - requires taking the limit $g^{2} e^{-\phi_{0} / 2} \rightarrow \infty$ in such a way that the middle expression of eq. (16) need not vanish.) We see that conical singularities are only possible for a one-parameter locus of tensions within the $T_{+}-T_{-}$ plane. A key question then becomes whether or not there is a physical reason why solutions must be chosen having only conical singularities.

Must GGP solutions satisfy eq. 16 in order to make physical sense? Possibly not, although a definitive resolution of this question is not yet possible. We must ask: Can solutions having non-conical singularities be produced by any reasonable resolution of the 3-brane structure? There are two reasons for entertaining the possibility of solutions having more complicated singularities than conical in the 6D context.

- For supergravity the existence of other fields in the bulk - such as the dilaton and other superpartners of the metric - can complicate the singularity structure since these other fields can themselves diverge as the brane position is approached [56].

- For cosmic strings in 4D the assumption of weak gravitational fields plays an important role in concluding that the external geometry is flat, and this need not be true for 3-branes in 6 dimensions.

The establishment of whether or not all of the general GGP solutions can be sourced by physically reasonable branes is clearly an important issue to resolve for deciding whether or not a hidden tuning has been slipped into the SLED proposal, and is currently under active study.

\section{D Self-Tuning II: Response to Tension Changes}

There is another way for which the properties of the GGP solutions can help understand whether or not 6D supergravity really provides a dynamical mechanisms for relaxing the effective $4 \mathrm{D}$ cosmological constant. In particular - as was first discussed in ref. [29], and again in more detail in [31 $]^{13}$ - if successful the SLED proposal must ultimately explain why the small effective 4D cosmological constant seen by brane observers is robust against arbitrary changes to the various brane tensions, such as might occur due to phase transitions taking place on the branes.

Imagine, then, we start off with one of the GGP geometries, perhaps one having only conical singularities, for which brane observers experience a flat 4 dimensions. Suppose that at time $t=0$, one of these tensions, say $T_{-}$, is instantaneously locally perturbed to a new value, $T_{-}^{\prime}$, and then held fixed and that the other tension, $T_{+}$, does not change during this process. How does the bulk geometry respond?

An honest calculation of this response requires a fully time-dependent treatment, because causality guarantees that the influence of the tension change can only propagate into the bulk at the speed of light. Our interest is in the final state towards which this transient evolution heads, and it is easiest to think about these alternatives by thinking of the $6 \mathrm{D}$ theory as a complicated $4 \mathrm{D}$ theory consisting of a great many massive Kaluza-Klein modes, $\Phi_{k}$, whose vacuum values are determined by a complicated multi-dimensional scalar potential, $V_{K K}\left(\Phi_{k}\right)$, which could be computed (in principle) by dimensional reduction. Our initial GGP solution may be regarded as being a local minimum of this

\footnotetext{
${ }^{12}$ For all of the GGP solutions the 2D metric in polar coordinates centered at a brane can be written in the form $\mathrm{d} r^{2}+c r^{p} \mathrm{~d} \varphi^{2}$ as $r \rightarrow 0$, for constants $c$ and $p$. The singularity at the brane position is only conical if $p=2$, in which case $c$ is related to the defect angle at the cone's apex [31].

${ }^{13}$ See also ref. [57].
} 
potential at which $V_{K K}=0 .{ }^{14}$ Since this potential depends on the values of the brane tensions it is perturbed once these tensions are adjusted, and the time-dependent evolution describes the rolling of the various KK modes towards their new minima. There are essentially three possibilities which are possible for the endpoint of this time-dependent evolution:

1. Successful Self-Tuning: In this picture, the endpoint of the transient evolution is another static geometry, for which the intrinsic 4D brane geometry is flat. In this case the evolution ends at a new local minimum of $V_{K K}$ which also vanishes, such as would occur if the new minimum corresponded to another GGP solution.

2. Non-catastrophic Adjustment: In this picture, the endpoint of the transient solution is another geometry for which the internal 2 dimensions are locally stable (modulo the flat direction), but for which $V_{K K}$ has been perturbed to be nonzero. In this case we would expect there being another solution to the $6 \mathrm{D}$ field equations corresponding to always being in this new 2D geometry, but with the 4 dimensions being described by de Sitter or anti-de Sitter space.

3. Catastrophic Runaway: A final option is a runaway, wherein the time-dependent evolution might shrink the 2D geometry to a point, or inflate it to infinite size. From the 4D perspective this would correspond to $V_{K K}$ developing a new unstable direction, along which the field can roll without stopping. Notice that this runaway option cannot arise (at the classical level) as a destabilization of the original flat direction, since the flatness of this direction is always guaranteed by the underlying scale invariance at the classical level now being discussed.

Although the results of a full time-dependent analysis are not yet available, some conclusions can be drawn given the existence of the general GGP solutions. In particular, Option 2 above can be ruled out for time evolution which does not break the initial axial symmetry of the extra dimensions. If the endpoint were towards a locally stable, axially symmetric $2 \mathrm{D}$ geometry having nonzero $V_{K K}$, then we'd expect there to also exist solutions to the $6 \mathrm{D}$ equations corresponding to eternally living at this 2D geometry, with the intrinsic 4-geometries being either de Sitter or anti-de Sitter spaces. But the GGP results show that such solutions do not exist, allowing this option to be ruled out.

It is also clear that since time evolution is continuous, the topological constraints (monopole number and Euler number satisfied by the initial configuration are guaranteed to remain preserved during the transient evolution. Of course, the particular expressions of these constraints as functions of the parameters of the initial solutions might change, since the new solutions might not satisfy the earlier assumptions under which the consequences of the topology was computed (such as by not being axially symmetric). But the topological nature of the constraints ensures that they automatically remain satisfied by the final solutions, as well as the time-dependent solutions which lead to them. Similarly, conserved quantities like energy and angular momentum must also remain unchanged during the timedependent evolution.

It is clearly of interest, then, to know if solutions within the GGP class exist for any choice of topological flux and tensions, since if such a solution were to exist then we might reasonably expect that the endpoint of the time-dependent evolution would be towards this static solution. This would correspond to Option 1 above, because the various KK modes would all settle down into new minima of their potentials at a point where $V_{K K}=0$ by virtue of the 4D flatness of all of the GGP solutions.

Given our present knowledge, we can say something about whether or not GGP solutions exist for pairs of tensions $T_{-}^{\prime}$ and $T_{+}$, at least for those which are small perturbations of an already existing GGP geometry. We can do so because it is possible to compute the brane tensions and magnetic flux of the source fields as functions of the 3 parameters of the GGP solutions which survive once the two scaling relations are used to set an additive constant in the dilaton, $\phi_{0}$, and the $U(1)_{R}$ coupling $g$ to specific values. A calculation of the Jacobian of these expressions shows that they are invertible, and so a choice of GGP parameters exists for any pair of tensions in the neighborhood of a given GGP solution [31]. In this way we see that it is plausible that the endpoint of the transient evolution could be towards another GGP solution, for which the 4D geometry is flat.

Clearly the endpoint of the evolution of the solutions once their source tensions are changed can only be properly resolved by constructing explicitly time-dependent solutions to 6D supergravity. Such a construction is underway, and so a definitive answer should soon be at hand [58]. In the meantime one might ask, why work so hard to answer this question in 6 dimensions at all, when the important part of the analysis could perhaps be done within a simpler 4D effective treatment?

\footnotetext{
14 More properly, a GGP (or any other classical) solution corresponds to a point along a flat trough of this potential, due to the existence of the marginal flat direction which is always guaranteed at the classical level by the classical scale invariance of the 6D supergravity equations.
} 
A 4D analysis of this type has been performed [57], which studies self-tuning in 6 dimensions, both for theories with and without supersymmetry, leading to the claim that the runaway solution (Option 3 above) is the one chosen by the theory. For the non-supersymmetric case this claim supports earlier, more detailed, studies [59] who also find difficulties with self-tuning within the non-supersymmetric context. It also agrees with the arguments given above inasmuch as the required underlying classical scale invariance is absent in the various non-supersymmetric $6 \mathrm{D}$ proposals. But does this analysis also rule out self-tuning within $6 \mathrm{D}$ supergravity, where such a scale invariance certainly exists? In this section it is argued that the calculations of ref. [57] are insufficient to tell.

The key assumption of the quantitative part of their analysis is the ansatz is to truncate the full $6 \mathrm{D}$ equations on the rugby-ball solutions to 4 dimensions, to restrict attention to two particular fluctuations about this solution: the time-dependence of the extra-dimensional volume, $a=e^{\psi(x)}$, and the 6D dilaton, $\phi=\phi(x)$. The background Maxwell field also is taken proportional to the 2D volume form, $\omega$, according to $F=b(x) \omega$. The truncation to these variables is done by writing

$$
\mathrm{d} s^{2}=e^{-2 \psi(x)} g_{\mu v}(x) \mathrm{d} x^{\mu} \mathrm{d} x^{v}+e^{2 \psi(x)} \mathrm{d} s_{2}^{2},
$$

where $d s_{2}^{2}=\mathrm{d} \theta^{2}+\lambda^{2} \sin ^{2} \theta \mathrm{d} \varphi^{2}$ is the line-element on the rugby-ball, whose defect-angles are related to $\lambda$. Such an ansatz seems to plausibly capture the relevant physics, and it is much easier to follow the time-dependence within the truncated action obtained by following only these modes (and the 4D metric), whose form is

$$
S=c \int d^{4} x \sqrt{-g}\left[R(g)-(\partial \phi)^{2}-4(\partial \psi)^{2}-V_{\alpha}(\phi, \psi)\right],
$$

for some constant $c$ and scalar potential $V$. The conclusions drawn are based on the properties of the solutions to the 4D equations which follow from this action. ${ }^{15}$

The veracity of these conclusions rests on the assertion that the solutions to the $4 \mathrm{D}$ theory of eq. 18 capture the same physics as the solutions of the full 6D theory, and there are two arguments which might (at first sight) indicate that this kind of reasoning might be true. First, it is known that truncation on the rugby ball is a consistent truncation of the 6D field equations [52], inasmuch as any solution to the 4D field equations obtained by truncating the 6D action using the rugby-ball solution is also automatically a solution of the full 6D field equations. The problem with such a truncation is that it says nothing about the stability of the solution obtained. For instance the truncation $X=0$ is a consistent truncation of the potential

$$
V(X, Y)=A(Y)-B(Y) X^{2}+C(Y) X^{4},
$$

in precisely the same sense, even if $B(Y)>0$. However we know that in this simple case $X=0$ is a local maximum of the potential rather than a minimum, and so the existence of the truncated solution $X=0$ provides poor guidance when trying to infer the endpoint of a time-dependent problem involving both of the fields $X$ and $Y$. In the 6D example the fields $X$ correspond to the various Kaluza Klein modes, which are typically nonzero in the known GGP solutions.

The second reason to trust a truncation to 4 dimensions rests on a firmer footing, since such a truncation is justified as the leading term in a low-energy expansion provided only that the truncated variables (i.e. $X$ in the above example) are systematically heavy compared with the variables which are kept (i.e. $Y$ in the above). Since this energy is measured relative to the local ground state, this criterion does not mistakenly keep unstable modes as in the above toy example. Furthermore, in the 6D example, one combination of the two fields kept in the 4D truncation (the dilaton and radion) is massless, and so can be understood in such a low-energy theory. Unfortunately, the other combination, $t=r^{2} e^{\phi}$, is massive and its mass is known to be precisely the same as the lowest nontrivial Kaluza Klein states [52, 60]. It follows that in order to reliably follow the dynamics of the radion, $\psi$, and the dilaton, $\phi$, separately in an effective 4D theory, one must include these Kaluza-Klein states in the low-energy theory as well, and cannot simply truncate them to zero by hand, as was done in [57]. ${ }^{16}$

\footnotetext{
15 These arguments have a historical precedent, since they closely resemble those made in the 1980's which seemed to indicate 6D solutions with de Sitter and anti-de Sitter 4-geometries were possible, such as when the background magnetic flux has monopole number not equal to \pm 1 . Part of the surprise with the solutions of ref. [50] was that this expectation was false, since the resulting geometries turn out to be both squashed and warped, but with flat intrinsic 4D geometries.

${ }^{16}$ See also the appendix of ref. [31] for a discussion of ref. [57].
} 


\section{Quantum Bulk Contributions: Why There is Something and Not Nothing}

The previous sections discussed at length the present status of the classical adjustment of bulk fields to cancel the brane contributions to the effective 4D cosmological constant. This section picks up the thread of the main story by returning to the size of the quantum part of the integration over bulk degrees of freedom. Given the cancellation between classical bulk physics and brane contributions, it is this bulk quantum contribution which is the net nonzero contribution to the observed Dark Energy.

To start: a summary of the result [27, 29, 61, 62]. In a nutshell, the upshot of this discussion is that the effective 4D vacuum energy obtained within 6D supergravity is similar to what might be expected given what is known about the vacuum energy within 4D supersymmetric models. The vacuum energy in a generic 4D supergravity is typically of order $\Delta V_{0} \sim m_{s b}^{2} M^{2}$, where $m_{s b}$ denotes the supersymmetry-breaking scale and $M$ is a larger scale (often the Planck mass). The suppression of $\Delta V_{0}$ by a power of $m_{s b}^{2}$ is easily understood because bose-fermi cancellations require the result to vanish in the limit that $m_{s b} \rightarrow 0$ [21]. For some models the result can be smaller, with $\Delta V_{0} \sim m_{s b}^{4}$, such as if special cancellations should ensure the vanishing of the $\mathscr{O}\left(m_{s b}^{2}\right)$ term. Essentially the same result holds for 6D supergravity, with the KK mass splitting, $m_{K K} \sim 1 / r$, playing the role of $m_{s b}$ and the 6D gravity scale, $M_{g}$, playing the role of $M$. While for generic 6D supergravities the 4D vacuum energy is of order $M_{g}^{2} / r^{2}$, there are some for which this leading result cancels to leave a subdominant result which is of order $1 / r^{4}$. Most interestingly, it is the supergravities whose 6D field content is that which arises by dimensional reduction from higher dimensions which appear to enjoy this kind of cancellation. Now to the details.

\section{Bulk Loops I: UV Sensitivity}

Since the issue of technical naturalness hinges on the dependence of the bulk-loop contribution on the heavy (TeV) scale, $M$, the focus of this first section is on the integration over bulk modes having energies of order $M$. (The contribution of the lighter fields is the topic of a later section.) At first sight it is tempting to think of this calculation as a particularly complicated 4D calculation which involves an enormous number of 4D (KK) modes. According to this point of view one would expect each KK mode to produce the usual 4D $M^{4}$-dependence in the vacuum energy, and then expect this result to just get worse once the sum over all of the KK modes is performed. This turns out to be a misleading way to calculate, since it hides simplifications which arise due to important constraints in the 6D theory (like 6D general covariance and locality) which constrain the contribution of all modes for which $M \gg m_{K K} \sim 1 / r$. These simplifications are better seen if the vacuum energy is directly computed within 6 dimensions, which is the point of view followed here. As usual, you can use any variables you wish to solve a problem, but if you use the wrong ones you'll be sorry.

In 6 dimensions, if $M \gg 1 / r$ then the most $M$-dependent contributions to the loop-generated vacuum energy can be written in terms of local interactions in the bulk, and on the branes [63]. The brane contributions arise from integration over bulk fields, because the existence of the branes introduces new kinds of UV dependence into the bulk [64]. This is how short-wavelength bulk fields 'know' about the presence of the branes, but because of the short wavelength of the modes involved these effects cannot reach further than a distance $d=\mathscr{O}(1 / M)$ into the bulk, and so only generate UVsensitive effective interactions at the brane positions. The potentially dangerous contributions to the vacuum energy are any of those which are proportional to positive powers of $M$, and so it is on those we focus.

For instance, the six-dimensional effective interactions which can be generated at one loop depending on positive powers of $M$ in chiral, gauged 6D supergravity have the form $\Delta S_{\text {eff }}=\Delta S_{B}+\sum_{b} \Delta S_{b}$, where [29, 61]

$$
\begin{aligned}
\Delta S_{B} & =\int d^{6} x e_{6}\left[c_{0} M^{6}+M^{4}\left(c_{1} R+c_{1}^{\prime} e^{\phi}+c_{1}^{\prime \prime}(\partial \phi)^{2}+\cdots\right)+M^{2}\left(c_{2} R^{2}+c_{2}^{\prime} e^{\phi} R+\cdots\right)\right] \\
\Delta S_{b} & =\int d^{4} x e_{4}\left[M^{4}\left(d_{0}+d_{0}^{\prime} e^{\phi}+\cdots\right)+M^{2}\left(d_{1} \mathscr{R}+\cdots\right)\right]
\end{aligned}
$$

where $R$ is the curvature scalar of the 6D metric, $\mathscr{R}$ is the same for the induced 4D metric, and so on. The various constants, $c_{i}, c_{i}^{\prime}, d_{i}$ etc., are dimensionless and the contribution to them due to any one 6D field is of order the 6D loop factor $1 /(4 \pi)^{3}$. For the coefficient of any fixed power of $M$, the ellipses describe other terms involving the other fields of $6 \mathrm{D}$ supergravity involving the same number of derivatives of these fields as appear in the explicitly-written powers 
of $R$. They also contain terms obtained by replacing factors of $R \sim 1 / r^{2}$ with factors of ${ }^{17} e^{\phi} \sim 1 / r^{2}$, the replacement of $R^{2}$ by other quadratic curvature invariants, and so on.

Some of these terms are easily disposed of. First, since the brane terms are similar to those which were already generated by integrating out the brane fields, they can be cancelled by the bulk classical response in precisely the same way. ${ }^{18}$ Next, it is $6 \mathrm{D}$ supersymmetry which disposes of the bulk cosmological constant (the $M^{6}$ term), since the contributions of bosons and fermions explicitly cancel in this term, as they must because local supersymmetry precludes the existence of a $6 \mathrm{D}$ cosmological constant. Similarly, it happens that the $M^{4}$ terms also cancel amongst the elements of a 6D supermultiplet, at least within the explicit calculations done so far [61], for which supersymmetry breaks in the bulk due to the background gauge flux not being in the $U(1)_{R}$ symmetry direction. Even if these $M^{4}$ terms did not cancel, they would not be dangerous inasmuch as they are simply renormalizations of the classical $6 \mathrm{D}$ action, and such a renormalization does not affect the classical self-tuning arguments described above. It does not because the precise value of the classical couplings (like the 6D Newton constant) is not important for the cancellation of brane tension and bulk curvature.

We see from this that the only potentially dangerous terms in $S_{\text {eff }}$ are the $M^{2}$ terms, and as we shall see these play a role which is very similar to the $m_{s b}^{2} M^{2}$ terms in 4 dimensions, which are also quadratic in the large massscale. Furthermore, explicit one-loop field-theoretic calculations [61] show that the coefficients of these terms do not vanish in all 6D supersymmetric field theories. However, recent calculations, which are still in progress, appear to show that $6 \mathrm{D}$ supergravities also exist having a field content for which these $M^{2}$ terms also vanish at one loop. For compactifications on Ricci-flat spacetimes in particular, it appears that the field content obtained by dimensionally reducing from 10 to 6 dimensions provides a particularly interesting example for which these $M^{2}$ terms cancel.

Higher Loops. Having the $M^{2}$ terms vanish at one loop is wonderful, but is it good enough? In particular, what of the contributions of higher bulk loops? Happily, it is possible to see why these higher loops need not be dangerous, at least for the gauged, chiral supergravities [29]. To see how this works, recall that in these theories the field equations imply that the background dilaton is related to the extra-dimensional radius by $e^{\phi} \sim 1 /(\mathrm{Mr})^{2}$, where the $6 \mathrm{D}$ gravity scale $M$ is here made explicit. But each loop involving bulk particles introduces additional powers of $e^{\phi}$, since the dilaton counts loops in 6D supergravity in the same way that it does in higher-dimensional supergravities.

So the large- $M$ contributions in 6 dimensions obtained at 2 and higher loops also involve the same powers of curvature and derivative as do the 1-loop contributions, eqs. 20, but pre-multiplied by additional powers of $e^{\phi} \sim 1 /(M r)^{2}$. We see that this extra loop factor is sufficient to tame the overall $M^{2}$ which pre-multiplies the curvature squared term, leaving a result which no longer involves a positive power of $M$. Only the one-loop-generated curvaturesquared terms are dangerous because of the extremely small size of the bulk coupling, $e^{\phi}$.

One might worry that there may be phenomenological difficulties with having such tiny bulk couplings, and this would indeed be true if the brane couplings were also this small since these brane couplings would include the observed strong and electroweak interactions. In general, quantities like the brane gauge couplings (in the Einstein frame) are expected to have an expansion in powers of $e^{\phi}$, as does the brane tension in eq. [20, of the form

$$
g_{b}=g_{0}+g_{1} e^{\phi}+\cdots,
$$

and the viability of phenomenology on the brane requires $g_{0} \neq 0$. This last condition is likely to make it more challenging to find brane types with the desired properties within string theory.

\section{Bulk Loops II: Weinberg's Theorem Revisited}

It is worth pausing at this point to revisit the issue of how these quantum corrections relate to Weinberg's No-Go theorem. Recall from the discussion of earlier sections that Weinberg's No-Go theorem states that if a scale invariant theory has a flat direction, then scale invariance cannot in itself keep this flat direction from being lifted by quantum effects (even if the scale invariance has no anomaly, and is a bona fide quantum symmetry). How does this relate to what has been found about quantum effects in SLED?

\footnotetext{
${ }_{17}^{17}$ Keep in mind here that the classical field equations for chiral, gauged 6D supergravity imply $e^{\phi} \sim 1 / r^{2}$ for rugby-ball solutions.

${ }^{18}$ It is possible that the curvature- and dilaton-dependence on the brane which is generated in this way is responsible for the non-conical singularities which arise in the GGP solutions.
} 
Weinberg's argument is correct when it says that the classical flat direction is lifted, as is explicitly seen above. The Casimir energy generates a potential for the classical flat direction, which can be taken to be parameterized by the extra-dimensional radius $r$. (Since for SLED the scale invariance is not a quantum symmetry, these corrections need not be so simple as being quartic in the appropriate field.) Weinberg's Theorem does not say how big these corrections must be, but in a supersymmetric theory it is natural to expect them to be set by the relevant supersymmetry-breaking scale.

What is new to the SLED picture is the decoupling it allows between the supersymmetry-breaking scale of the bulk from that on the brane [40]. But since the flat direction involves only bulk fields (like $r$ ) it is only the supersymmetry breaking scale in the bulk which is relevant for the lifting of the flat direction. Crucially, Weinberg provides no argument against this separation of supersymmetry-breaking scales.

\section{Bulk Loops III: Massless 6D Modes}

Although quantum bulk contributions to the vacuum energy are nonzero, they do not generate a cosmological constant. Rather, the Casimir energy which is produced describes a scalar potential for the flat direction of the classical theory. Understanding this potential is a crucial step towards understanding the dynamics which explains why the size of the extra dimensions is as large as we require, $1 / r \sim 10^{-2} \mathrm{eV}$. In this section it is shown what this potential looks like, and that the mass of this would-be massless field is sufficiently light as to be cosmologically rolling today. This implies that within the SLED proposal the dynamics generated for $r$ by the Casimir energy predicts a time-dependent Dark Energy, with a particular form for the Dark Energy potential. Furthermore, SLED automatically explains why the small mass of the radion field is technically natural in addition to why the value of the Dark Energy density is naturally small, using the mechanism first enunciated in refs. [11, 25].

The total one-loop Casimir energy is obtained by adding the local contributions, discussed above, of the very massive modes to the contribution of the lighter modes coming from those fields which are massless in the 6D sense. The contributions of these lighter modes have been known for some time for various 6D theories dimensionally reduced on spheres and torii and in the absence of branes [65, 66]. More recent calculations (some of which are in progress) generalize these to include the branes and their back-reaction, and give similar results [67, 61, 62].

For instance for the rugby-ball compactifications of chiral, gauged 6D supergravity $m_{s b} \sim 1 / r$ is the supersymmetrybreaking scale of the bulk sector (in the 4D Jordan frame), and the resulting vacuum energy is the Casimir energy obtained by integrating out bulk loops. The result is a potential for the radion, $V(r)$, which expresses the quantum lifting of the classical flat direction: ${ }^{19}$

$$
V(r)=\frac{c_{2} M^{2}}{r^{2}}+\frac{c_{3}}{r^{4}}\left[\log \left(M^{2} r^{2}\right)+C\right] .
$$

Here $M \sim M_{g}$ is the $\mathrm{TeV}$ scale associated with the integration over heavy fields, and the dimensionless constants, $c_{2}$ and $c_{3}$, are calculable given the field content of the $6 \mathrm{D}$ supergravity, and are related to the average over the 2 extra dimensions of those appearing in $S_{B}$ in eqs. (20). Unlike the logarithmic term, the constant $C$ depends on the precise matching conditions used at the higher scale $M$. The logarithmic term arises due to the renormalization of higher-dimensional effective interactions like $R^{3}$, and higher powers of $\log r$ can be expected at higher-loop orders. The coefficient $c_{3}$ can be explicitly computed for a given choice of massless $6 \mathrm{D}$ multiplets, and once integrated over the volume of the 2 extra dimensions is typically of order $1 /(4 \pi)^{2}$. By contrast, a calculation of the constants $c_{2}$ and $C$ depends on the details of the spectrum of the theory at the electroweak scale, $M$, and of particular interest are those theories for which this spectrum ensures $c_{2}=0$ [61].

In order to understand the dynamics of the model it is necessary to also include the 4D Einstein and kinetic terms for $r$ in the low-energy lagrangian [11]. These lead to the canonical scalar, $\varphi$, which is related to $r$ by $M r=e^{k \varphi / M_{p}}$, for a positive constant $k$ whose numerical value is not important for the present purposes. In terms of $\varphi$ the scalar potential, eq. (22), becomes

$$
V(\varphi)=M_{p}^{4}\left[\hat{c}_{2} e^{-2 k \varphi / M_{p}}+\hat{c}_{3} e^{-4 k \varphi / M_{p}}\left(C+\frac{\varphi}{M_{p}}\right)\right] .
$$

\footnotetext{
${ }^{19}$ For toroidal compactifications of the ungauged 6D supergravities, the potential has a slightly different form: $V\left(r, u^{i}\right)=W\left(u^{i}\right) / r^{4}$, where the $u^{i}$ denote the various shape moduli and other related scalar fields under supersymmetry. In particular, no logarithmic terms are generated in this case at one loop 65,62$]$.
} 
Here the constants $\hat{c}_{i}$ are proportional to the $c_{i}$ appearing in $V(r)$.

Suppose now that we specialize to the present epoch, for which $r=r_{0} \sim 10 \mu \mathrm{m}$. Then if $c_{2} \neq 0$, using $M_{p}=M^{2} r_{0}$ shows that the Dark Energy density is of order $V \sim M^{2} / r_{0}^{2}$ and the $\varphi$ mass is of order $m_{\varphi} \sim 1 / r_{0}$. Both of these are too large to provide an acceptable phenomenology for the Dark Energy. But the models of interest have $c_{2}=0$, as is required to ensure a sufficiently small value for $V: V \sim 1 / r_{0}^{4}$. As is clear from the above, this condition $\left(c_{2}=0\right)$ automatically also ensures a sufficiently small scalar mass, $m_{\varphi} \sim 1 /\left(M_{p} r_{0}^{2}\right) \sim H_{0}$, to allow a time-dependent dark energy during the present epoch.

SLED therefore leads to a particular type of time-dependent Dark Energy having a specific kind of modified exponential potential. It also predicts that the relevant scalar mass for the late-time cosmology is of order $m_{\varphi}^{2} \sim V_{0} / M_{p}^{2}$, which allows the smallness of this mass to be ensured given that the present-day radius, $r_{0}$, is large enough to permit $V$ to be observably small (more about why $r_{0}$ might be this small below).

\section{Embedding into More Fundamental Theories?}

Part of the appeal of the SLED proposal is that it involves supergravity and branes, and so it can plausibly be embedded into what is at present probably our best-motivated fundamental theory of physics on the shortest scales: string theory. This section briefly digresses to summarize what is known about the possibility of doing so in more detail.

A successful embedding of SLED into string theory consists of identifying string vacua for which the low-energy excitations describe both the 6D supergravity of the bulk and the degrees of freedom trapped on the branes. Indeed, the non-chiral (gauged and ungauged) 6D supergravities have a known string provenance in this way as dimensional reductions of 10D string vacua. A complete string derivation of the chiral 6D theory is not yet known - see however [68] - although it is plausibly derivable as the low-energy limit of $10 \mathrm{D}$ heterotic string theory compactified on $\mathrm{K}_{3}$ [69].

Knowing how the branes arise within string theory is also important, and this has several different aspects. It involves finding string vacua having 3-branes in the effective low-energy 6D supergravity; it involves finding the particles of the Standard Model living on one of them; and it involves obtaining brane-bulk couplings which are phenomenologically acceptable [27, 28]. A complete understanding of all of these issues is likely to require an understanding of the origin of the corresponding $6 \mathrm{D}$ bulk supergravity as a precondition. Once the higher-dimensional origin of the 6D supergravity is known, the kinds of branes which are available to play the role of the 3-branes in the effective theory may begin to be addressed. Since compactifications of Type IIA and Type IIB supergravities in 10D are known to produce the non-chiral, ungauged theories in 6 dimensions, the effective 3-branes in these theories could be obtained as D3 branes or as higher-dimensional D-branes wrapped about cycles in the 4 dimensions between 10 and 6 . Alternatively, if the chiral 6D supergravity should emerge as expected as a compactification of $10 \mathrm{D}$ heterotic supergravity on $\mathrm{K} 3$, then the 3-branes might instead emerge as NS5-branes wrapped on 2-cycles in K3.

An important difficulty which any such brane construction must confront is the large hierarchy between the size of the gauge couplings on the brane and those in the bulk. That is: why should $g_{0} \neq 0$ in eq. (21)? A discussion of some of the other issues involved in embedding SLED into a string framework is given in ref. [30].

\section{Observational Implications}

Because the SLED proposal modifies physics at sub-eV energy scales, it has many other observational implications besides its explanation for the small size of the cosmological constant. This section very briefly summarizes some of these, including implications for cosmology, tests of gravity on short and long distances, and implications for particle physics (including observable signals at up-coming colliders like the LHC). The purpose of this section is to summarize some of these other predictions.

What emerges from this summary is that SLED is unique among Dark Energy proposals in several ways. First, no other Dark Energy theory has such wide-ranging implications outside of cosmology, because no other proposal purports to address the underlying naturalness issues which require changing physics in the energy range from $10^{-2} \mathrm{eV}$ to several TeV. Second, determining the size of the extra dimensions requires keeping track of order-one contributions to the vacuum energy, such as the factors of $2 \pi$. This is a huge improvement over the theoretical uncertainties of usual Dark Energy proposals, which can easily involve factors of $10^{50}$. Even more interestingly, the extra-dimensional sizes 
to which the Dark Energy leads are close to the present observational bounds on extra-dimensional sizes. Best of all, the SLED connection between the Dark Energy and extra-dimensional size makes it very predictive and falsifiable, since it is not possible to make the extra dimensions smaller — and so easier to hide — without destroying the explanation of the observed Dark Energy.

\section{Bounds on Extra-Dimensional Radii}

This section discusses the most restrictive observational limits on the possible size the extra dimensions can take. The strongest such limit comes from the constraint that the energy loss into Kaluza Klein modes does not provide too efficient an energy-loss mechanism for supernovae [34, 35]. This process has been studied in detail for the special case of the radiation of gravitons into the bulk, with the result [35] that an acceptably small energy-loss rate requires the 6D gravitational scale, $M_{g}$, to satisfy $M_{g} \gtrsim 8.9 \mathrm{TeV}$. For unwarped extra dimensions this requires the extra-dimensional size to be $r \lesssim 10 \mu \mathrm{m} .{ }^{20}$ This limit could be even stronger for SLED, because there are more states in the bulk into which energy may be lost. Not all of these may have a nonzero amplitude for single-particle emission, however, and so the precise loss rate requires a more detailed calculation. A crude estimate is

$$
\Gamma_{S L E D} \approx \Gamma_{L E D} \mathscr{N},
$$

where $\mathscr{N}$ is an estimate of how many more channels are available in the bulk, relative to the purely gravitational signal of the nonsupersymmetric case. This leads to the bound

$$
M_{g}>\mathscr{N}^{1 / 4} 8.9 \mathrm{TeV} .
$$

In the worst-case scenario where all modes can be radiated we might have $\mathscr{N} \sim \frac{1}{9}\left(32+16 N_{g}+8 N_{m}\right)$, where as before $N_{g}$ and $N_{m}$ count the number of gauge and matter multiplets in the bulk. For plausible choices for $\mathscr{N}$ this leads to a constraint on $r$ which is only marginally stronger than the LED constraint: $M_{g} \gtrsim 12 \mathrm{TeV}$ and $r \lesssim 5.3 \mu \mathrm{m}$.

There are also other, nominally stronger, bounds on extra-dimensional models which come from the non-observance of Kaluza-Klein modes decaying into photons after having been produced in supernovae or in the early universe. We ignore these bounds for the present purposes, since unlike the bound just discussed they can be completely evaded depending on the details of the model. For instance, they do not arise if KK modes can efficiently decay into invisible light modes on other branes. We regard the model building which such an evasion requires to be well worth the cost if the resulting theory can make progress on the much more difficult cosmological constant problem.

\section{Post-BBN Cosmology}

Besides providing a natural size for the Dark Energy density, the SLED proposal makes many further predictions concerning the properties of Dark Energy. First and foremost, it predicts that the Dark Energy is dynamically evolving in time even now, and so is not simply a cosmological constant. Furthermore, it accounts for the amount of Dark Energy and the very light mass required by its present-epoch evolution in a way which appears to be technically natural. Within SLED the evolving 4D scalar field (or fields) have a microscopic origin as the overall breathing mode of the 2 large extra dimensions, $r$ (plus possibly various shape moduli for these same dimensions).

Dark Energy. Because of this microscopic picture SLED predicts a very specific form for the Dark Energy field's scalar potential. It must be the potential of the Casimir energy for the appropriate 6D fields in the presence of a particular extra-dimensional shape. Because the extra dimensions are large, the result is robustly a series in inverse powers of $r$, often with logarithmic corrections, which is arranged to start at order $1 / r^{4}$. (It is the values of the constants in this potential which depend on the 6D field content and the details of the extra-dimensional shape.)

More precisely, within SLED models the Dark Energy arises as a Casimir energy in the presence of branes within a compact two-dimensional solution to 6D supergravity. As discussed above, for the rugby-ball solutions this Casimir

${ }^{20}$ Ref. [35] quotes $r<1.6 \mu \mathrm{m}$, but uses conventions where the extra-dimensional volume is given by $V=(2 \pi r)^{2}$ rather than $V=r^{2}$. 
energy is given in the Einstein frame by

$$
V(r)=\frac{A}{r^{4}}\left[1-a \log \left(M_{p} r\right)+\frac{b}{2} \log ^{2}\left(M_{p} r\right)+\cdots\right]+\mathscr{O}\left(\frac{1}{r^{6}}\right),
$$

where $A, a$ and $b$ are dimensionless constants and the ellipses represent possible terms involving higher powers of $\log r$. Because terms like $\log ^{\ell} r$ first arise at $\ell$-loop order, within the SLED framework it is natural to expect $b / a \sim a \sim N_{\text {eff }} /(4 \pi)^{3}$, where $N_{\text {eff }}$ provides some measure of the mismatch between bosons and fermions (and so depends on the details of how supersymmetry is broken). For instance, if taken to be of order the total number of bosonic states in 6D supergravity then $N_{\text {eff }}=16+8 N_{g}+4 N_{m}$, where $N_{g}$ counts the number of gauge multiplets and $N_{m}$ counts the number of hypermultiplets. As written, $V$ is positive for all $r$ provided $A>0$ and $2 b>a^{2}$.

The potential of eq. (26) is known as an Albrecht-Skordis potential, and is known to be able to describe a phenomenologically acceptable description of time-dependent Dark Energy [70, 11]. If $a^{2}+(b / 4)^{2}-2 b>0$ the potential has a local minimum at $r_{-}$and a maximum at $r_{+}$, where $4 b \log \left(M_{p} r_{ \pm}\right)=b+4 a \pm \Delta$, and $\Delta=4\left[a^{2}+(b / 4)^{2}-\right.$ $2 b]^{1 / 2}$. In these cosmologies the acceleration of the present-day Dark Energy is due to the evolution of $r$ becoming potential-dominated in the vicinity of $r_{ \pm}$, and so $r \sim r_{ \pm}$during the present epoch. Notice that $r_{+}$and $r_{-}$can be naturally of order $10 \mu \mathrm{m}$ provided $a / b \approx 70$. Remarkably, such values are not unreasonable for $6 \mathrm{D}$ Casimir energies given that for these $a / b \sim(4 \pi)^{3} / N_{\text {eff }}$.

The present-day value of the Dark Energy density in SLED then becomes

$$
\rho \approx V\left(r_{+}\right)=\frac{A}{16 r^{4}}[b+\Delta],
$$

and so the observed size for $\rho$ is controlled by the values of $A, a$ and $b$. Given estimates for these quantities one can determine the smallest value for $r$ which is consistent with Dark Energy observations. Using the reasonable values $A \approx 1$ and $b \sim a^{2}$ with $a \sim N_{\text {eff }} /(4 \pi)^{3}$, we see that for reasonable values of $N_{\text {eff }}, \rho$ can take the observed value $(0.003$ $\mathrm{eV})^{4}$ with $r$ close to its maximum allowed value $r \sim 5 \mu \mathrm{m}$ (up to within the uncertainties of the estimates given). Furthermore, $r$ cannot be much smaller than this value since this leads to much larger values for $\rho$ due to the scaling $V \propto 1 / r^{4}$.

Additional constraints also arise within SLED beyond those normally considered for Albrecht-Skordis potentials because of the extra-dimensional origin of the scalar fields involved. In particular, because the overall motion of $r$ implies a change in the value of Newton's constant over cosmological epochs, it cannot have changed by more than $\sim 10 \%$ between the epoch of nucleosynthesis (BBN) and now - a condition which can also be satisfied in these types of models [11]. Furthermore, the parameters of the potential must be chosen to ensure that the present-day potential-energy domination occurs for radii of order $r \sim 10 \mu \mathrm{m}$. Such large values are ensured if the coefficients of the logarithmic terms in the potential are of order $b / a \sim 1 / 70$, and remarkably such values arise naturally within SLED as loop factors.

Although more dedicated studies need to be done, it seems likely that SLED can share the viable phenomenology of the models examined in ref. [11]. If so, it is likely that the successful description of present-day cosmology depends on the particular initial conditions chosen for $r$ just before the BBN epoch. This would imply that a real understanding of the cosmology of Dark Energy is likely to require a better theory of its initial conditions, such as why $r \sim \mu \mathrm{m}$ should be already true at the BBN epoch. It must also explain why the various KK modes of the 2 dimensions should no longer be excited by the time the BBN epoch is reached, since these also can cause problems for late-time cosmology (more about this below). What is exciting is that the possibility of embedding the effective 6D SLED theory into a more microscopic framework, such as within string theory, is likely to provide this theory of initial conditions, such as by explaining them as a consequence of an earlier inflationary epoch [71].

Dark Matter. It is not yet known how Dark Matter might fit into the SLED picture. It would be attractive to be able to use a variant of the standard Weakly-Interacting Massive Particle (WIMP) paradigm to describe the Dark Matter as being the relic abundance of a stable particle having a weak-scale mass and a weak-interaction annihilation cross section. This paradigm is attractive because any such particle naturally has the presently-observed abundance provided only that it was originally in thermal equilibrium with the other observed particles at epochs when the temperature was of order their masses. For supersymmetric theories it is common to use the Lightest Supersymmetric Particle (LSP) as the Dark Matter WIMP, but this is harder to do for SLED models because the LSP is the gravitino, which is much too light. Furthermore, ordinary particles on our brane need not have superpartners at all because supersymmetry is realized nonlinearly there, removing at a stroke most of the standard Dark Matter candidates [45]. 
The WIMP proposal is nevertheless very attractive for the SLED proposal because of the 'Why Now?' problem of any model of time-dependent Dark Energy. This question asks why it should be that the universal energy density in matter, radiation and Dark Energy should happen to have been so similar to one another during the very recent universe. This question is all the sharper given that these three forms of energy vary very differently as a function of the universal scale factor as the universe expands. A clue as to how to understand this problem within the SLED framework comes from ref. [72], who argue that the Why Now? problem has a natural solution if: 1. The Dark Matter is a relic WIMP abundance, and 2. the Dark Energy is a cosmological constant which is parametrically of order $\rho \sim\left(M_{w}^{2} / M_{p}\right)^{4}$ (as is automatically true for the SLED proposal). In such a case, they argue, their present-day abundances would naturally be close to one another, since they are both set by ratios of $M_{w}$ and $M_{p}$.

Can WIMPS be found within SLED models? The complete answer is not known, although it is promising that there can an enormous number of weakly-interacting states available having masses at the weak scale, the decays of many of which can also be prevented by a conservation law. As remarked in ref. [30] there are KK modes associated with the compactifications from 10 dimensions down to 6 , as well as string modes or winding modes about these 'other' 4 dimensions, any of which could have the required properties [73]. However in their simplest forms all of these proposals require the relevant modes to be in thermal equilibrium at temperatures of order the $\mathrm{TeV}$ scale. As is discussed below, this assumption can be problematic in models having Large Extra Dimensions.

\section{Pre-BBN Cosmology}

It happens that in LED and SLED models the real problem with Dark Matter is not why there is so much of it, but why there is so little. This is because LED and SLED models can become dangerous if KK modes in the large 2 extra dimensions (as opposed to the smaller 4 weak-scale dimensions) should be stable and be in equilibrium not much before the BBN epoch. If so, then these modes can be too abundant and their energy density can contribute too much to the universal energy budget and either ruin the predictions of BBN, or make the universe matter-dominated too early in its history [74].

This problem needs careful study, but need not be generic because it depends on fairly model-dependent features of both the geometry of the extra dimensions and of the universe's thermal history. When considering LED models it is normally assumed that these modes were not in equilibrium when ordinary matter had temperatures above a 'normalcy' temperature, $T_{*}$, which must be higher than the BBN scale but not as high as the weak scale. Unfortunately, this option is not available if we wish to explain the Dark Matter abundance in terms of a thermally generated WIMP, as in the previous section.

A vulnerable assumption in these bounds is that some Kaluza-Klein modes are stable because they carry a conserved charge. This is true in the simplest compactification, such as for torii and sphere, but is not true when the 2 large dimensions are not symmetric, or because of the presence of branes (see for instance [75]). In these cases there need not be conserved charges in the bulk which the KK modes can carry. If so, then nothings precludes their quick decay into massless states which would not dominate the universal energy density. If this were done early enough it could also avoid ruining the constraints on excess radiation density at the BBN epoch. It is sufficient to have the extra dimensions be asymmetric at very early epochs, when the universe's temperature is above $T_{*}$, and this need not preclude their being very symmetric right now.

Indeed, this kind of evolution of the extra dimensions is quite likely to occur within the SLED proposal because its energy cost is not high when temperatures are as large as $T_{*}$. This is particularly true, given the propensity of the extra dimensions to become warped and squashed in response to changes in tension on the various branes. If so, a realistic calculation of the residual energy tied up in KK modes for the 'large' 2 dimensions must await a better understanding of the time-dependent geometries in 6 dimensions, which is currently under study.

\section{Tests of Gravity}

There are two sources of deviation from General Relativity which SLED models predict.

Short-Distance Tests. First, deviations from Newton's Law are expected on micron length scales, because these are the scales at which the relevant effective theory becomes 6-dimensional. The deviations from Newton's inverse-square law for gravitation must break down in SLED for the same reason as it does within the earlier LED proposal: the effects of ordinary graviton exchange begin to compete at these distances with the exchange of various Kaluza-Klein 
modes from the two large extra dimensions. The range over which the deviations from the inverse-square law arise is set by the lightest of the nonzero masses of the Kaluza-Klein modes. Although the precise value of this lightest mass depends on the details of the precise shape of the extra dimensions, we have seen above that they are of order $m_{K K} \sim 2 \pi \kappa / r$ with $\kappa=1$ for a toroidal compactification. It would be worthwhile computing the form of the predicted force law in more detail for representative geometries, given the many bulk fields in SLED whose Kaluza Klein modes could be relevant.

It is central to the SLED proposal that the understanding of the Dark Energy density fixes $r /(2 \pi)$ to like in the micron range, and so the absence of deviations from Newton's Law in this range would rule out the model. This range is some two orders of magnitude below the $100 \mu \mathrm{m}$ range to which the best present searches for these effects are sensitive [39], but are within reach to the next generation of proposals.

Long-Distance Tests. Second, the existence of very light 4D scalars (like $r$ or the any shape moduli) implies that the theory of gravity relevant right up to the present-day horizon size is a particular type of 4D scalar-tensor model. This follows quite generally as a consequence of the relaxation mechanism it predicts for the Dark Energy density, which we have seen leads naturally to one or more fields, $\varphi$, whose mass is presently incredibly small, $m_{\varphi} \sim H_{0} \sim 10^{-33}$ $\mathrm{eV}$. Unlike most other Dark Energy proposals, we have seen how this small scalar mass can be naturally stable against quantum effects within the SLED framework.

At the classical level the theory is very predictive because the breathing mode of an extra dimension is known to couple to ordinary matter on our brane through their contributions to the trace of the stress tensor, $T_{\mu}^{\mu}$. At first sight this seems to be a fatal prediction since gravitational-strength couplings of this form can already be ruled out with some precision. A more detailed look is again more interesting, and whether these tests falsify SLED cosmology relies on more detailed calculations of the $r$-field couplings than are presently available. That they do can be seen from models such as those of refs. [25, 11], for which the precise strength and coupling of the light field can actually depend on some of the details of its cosmological evolution, since they can evolve during the history of the universe. They can do so because in the models investigated these couplings are field-dependent. Since the measurements which constrain these models are performed only during the present epoch, they are satisfied provided the couplings happen to be small at present, and this is what happens in the cosmological models of [11].

Similar effects can arise within SLED scenarios because loop effects can introduce an $r$-dependence into otherwise field-independent quantities. Clearly the question of how big these couplings now are (and whether their motion can be traced within the domain of perturbation theory) can be addressed in a more focussed way given a real calculation of the potential and interactions of the field $\varphi$ (and any other light fields) from a specific microscopic model for the extra-dimensional geometry. This provides yet more motivation for exploring the details of such compactifications, including loop effects, more closely. We see that, unlike the short-distance tests, long-distance tests of gravity need not be fatal to SLED models if deviations from General Relativity on these scales are not seen, because the size of the present-day couplings of these light scalars is not central to the explanation of the Dark Energy properties.

The possibility that there can be very light scalar fields around providing us with interesting non-standard gravitational physics on the longest distance scales is particularly exciting given the recent new opportunities for testing this kind of scenario. It motivates, in particular, a more careful exploration of the phenomenology of scalar tensor theories for solar-system tests, as well as for tests in more exotic settings (like the recently-discovered system consisting of two pulsars which orbit one another [76]). Given a theoretical framework for such forces, it becomes possible to make more specific statements about the nature of their expected couplings, which allows more focussed analyses of the constraints which can be expected from these systems.

\section{Implications for Particle Physics}

Most remarkable of all are the consequences which SLED models have for particle physics, including most notably the likelihood of observable missing-energy signals at the Large Hadron Collider (LHC) which is currently under construction at CERN.

Collider Physics. A robust consequence of the SLED proposal is the existence of many bulk Kaluza-Klein modes whose mass spacing is of order $2 \pi / r \sim 0.3 \mathrm{eV}$. Although each of these modes is coupled to ordinary particles only with gravitational strength, their enormous phase space at $\mathrm{TeV}$ energies makes their collective effects enter into observables 
at collider experiments with rates of order

$$
\Gamma(E) \propto\left(\frac{1}{M_{p}^{2}}\right)(E r)^{2} \sim\left(\frac{E^{2}}{M_{g}^{4}}\right),
$$

whose size is controlled by powers of $M_{g}$ rather than $M_{p}$. For this reason the production of missing energy at $\mathrm{TeV}$ scale colliders is a robust signal of the SLED proposal.

Of course, the same arguments apply equally well to the non-supersymmetric LED proposal, where they again argue for a robust collider signal, and this has led to extensive studies of the possible phenomenological signatures of graviton emission into the bulk [77]. Since these studies typically show that observable effects require $M_{g} \lesssim 1 \mathrm{TeV}$, one might worry that the supernova constraint $M_{g} \sim 10 \mathrm{TeV}$ must preclude the expected SLED collider signal from being observably large.

We argue that this conclusion may be too pessimistic for several reasons. First, these calculations were done only for the graviton and we have already seen that radiation rates into the bulk are more efficient in SLED because of the existence there of all of the graviton's super-partners [32, 33]. Furthermore, the pessimistic conclusion also relies too heavily on there being no new physics at energies which are lower than the scale $M_{g}$, even though $M_{g}$ is really only the scale of 6D gravity as measured by the higher-dimensional Newton constant. As such, it need not be the threshold at which new physics first emerges. (A similar error would be made for the weak interactions if the Fermi constant, $G_{F}^{-1 / 2} \sim 300 \mathrm{GeV}$, were used to infer where the scale where the physics of the electro-weak interactions first appears. In reality we know that this physics starts at $M_{w}=80 \mathrm{GeV}$ rather than $300 \mathrm{GeV}$, because of the appearance of small dimensionless couplings in the relation between $G_{F}$ and $M_{w}$.) In the same fashion, it is likely that within the SLED proposal the string scale is at or below the scale $M_{g}$, making some states potentially available to experiment at scales of a few $\mathrm{TeV}$.

Furthermore, depending on how it arises within a more microscopic theory like string theory, there is a possibility in the SLED picture that there are also Kaluza-Klein and winding states associated with the 'other' 4 compact dimensions obtained when compactifying from 10 to 6 dimensions. As we saw in earlier sections, these should also have masses in the $\mathrm{TeV}$ regime provided the 4 small internal dimensions are not strongly warped. In this regime collider reactions should resemble string collisions near Planckian energies [78]. A more precise study of the phenomenology of these modes requires the study of the string compactifications from 10 to 6 dimensions which lead to the kinds of 6D models of present interest.

The most likely collider signal (besides the direct production of new string or KK states) of SLED physics is therefore likely to be missing energy, which can be produced in association with an isolated jet or lepton. (For a recent study of bulk-scalar emission at ATLAS see ref. [33].) Such a signal is unlikely to be confused with the missing energy signals of alternative proposals, such as the Minimal Supersymmetric Standard Model (MSSM).

Non-Accelerator Physics. SLED has tantalizing possibilities for neutrino physics given the large number of neutral fermions which naturally occur in the bulk, and which are required by supersymmetry to have vanishing 6D mass. Furthermore their KK masses are $m_{K K} \sim 1 / r \sim 10^{-2} \mathrm{eV}$, which puts them right in the region where neutrino masses are likely to be. The main obstruction to these models at present seems to come from astrophysical bounds, since the same interactions which can provide neutrino masses tend also to predict an overly abundant rate of energy loss into the extra dimensions in supernovae [79]. Study of the phenomenology of these systems is underway [80].

From all of the above it is clear that Supersymmetric Large Extra Dimensions have fascinating implications which seem to cut a wide swathe across cosmology, astronomy and physics in laboratories. The proposal relates the Dark Energy in a fundamental way to observable tests of gravity and to signals in particle colliders. If true, its verification will be spectacular. One can only hope!

\section{ACKNOWLEDGMENTS}

I thank the organizers of the Texas A\&M workshop for their hospitality as well as for their kind invitation for me to speak. The research described here was done together with numerous collaborators to whom I am indebted, including Yashar Aghababaie, Andy Albrecht, Georges Azuelos, Hugo Beauchemin, Jim Cline, Quim Matias, Susha Parameswaran, Finn Ravndal, Constantinos Skordis, Gianmassimo Tasinato, Fernando Quevedo and Ivonne Zavala C. 
I also thank Z. Chacko, G. Gibbons, G. Moore, C. Pope and C. Wagner for useful suggestions and criticisms of sixdimensional self-tuning. Finally I am obliged to Steven Weinberg, both for teaching me how to think about effective field theories and for impressing on me the seriousness of the cosmological constant problem.

\section{REFERENCES}

1. For reviews, see for example: V. Trimble, in the proceedings of the First International Symposium of Dark Matter in the Universe, Bel Air California, D.B. Cline editor, World Scientific (1994); L.M. Krauss, The Standard Model, Dark Matter and Dark Energy: From the Sublime to the Ridiculous, in the proceedings of the XIV Canary Islands Winter School in Astrophysics, 2002 astro-ph/0406673|.

2. S. Eidelman et al., Review of Particle Properties, Phys. Lett. B592, (2004) 1.

3. S. Perlmutter et al., Ap. J. 483565 (1997) |astro-ph/9712212|; A.G. Riess et al, Ast. J. 1161009 (1997) |astro-ph/9805201|; N. Bahcall, J.P. Ostriker, S. Perlmutter, P.J. Steinhardt, Science 284 (1999) 1481, astro-ph/9906463|; D.N. Spergel, et al. |astro-ph/0302209|.

4. $\quad$ S. Weinberg, Rev. Mod. Phys. 61 (1989) 1.

5. M. Milgrom, Ap. J. 270 365-370; 371-283; 384-389; for a more recent review, see also: R.H. Sanders and S.S. McGaugh, Ann. Rev. Astron. Astrophys. 40 (2002) 263-317, |astro-ph/0204521|.

6. G. Dvali, G. Gabadadze and M. Porrati, Phys. Lett. B485 (2000) 208-214 |hep-th/0005016|.

7. P.J.E. Peebles and B. Ratra, Rev. Mod. Phys. 75 (2003) 559-606 |astro-ph/0207347|.

8. P.J.E. Peebles and B. Ratra, Ap. J. 325 (1988) L17; Phys. Rev. D37 (1988) 3406; C. Wetterich, Nucl. Phys. B302 (1988) 668; E. J. Copeland, A. R. Liddle, and D. Wands, Ann. N. Y. Acad. Sci. 688 (1993) 647.

9. S.M. Carroll, contribution to the SNAP Yellow Book |astro-ph/0107571|; A.R. Liddle and R.J. Scherrer, Phys. Rev.D59 (1999) 023509; C.P. Burgess, in Cape Town 2002, Dark matter in astro- and particle physics, 274-284 [astro-ph/0207174|.

10. A. H. Guth, Phys. Rev. D23 (1981) 347; A. D. Linde, Phys. Lett. 108 (1982) 389; A. Albrecht and P. J. Steinhardt, Phys. Rev. Lett. 48 (1982) 1220.

11. A. Albrecht, C.P. Burgess, F. Ravndal and C. Skordis, Phys. Rev. D65 (2002) 123506 |astro-ph/0107573|.

12. K. Coble, S. Dodelson and J. A. Frieman, Phys. Rev. D55, 1851 (1997) |astro-ph/9608122|; R. R. Caldwell, R. Dave, and P. J. Steinhardt, Phys. Rev. Lett. 80 (1998) 1582 (1998) |astro-ph/9708069|; R. R. Caldwell and P. J. Steinhardt, Phys. Rev. D57, 6057 (1998) astro-ph/9710062|; P. G. Ferreira and M. Joyce, Phys. Rev. D58 (1998) 023503 |astro-ph/9711102|; E. J. Copeland, A. R. Liddle, and D. Wands, Phys. Rev. D57 (1998) 4686 |gr-qc/9711068|; I. Zlatev, L. M. Wang and P. J. Steinhardt, Phys. Rev. Lett. 82, 896 (1999) |astro-ph/9807002|; Phys. Rev. D59, 123504 (1999)|astro-ph/9812313|.

13. C. Armendáriz-Picón, T. Damour, and V. Mukhanov, Phys. Lett. B458, (1999) 219 |hep-th/9904075|; J. Garriga and V. Mukhanov, Phys. Lett. B458, (1999) 219 |hep-th/9904176|; M. Malquarti, E.J. Copeland, A.R Liddle and M. Trodden, Phys. Rev. D67 (2003) 123503 |astro-ph/0302279|; M. Malquarti, E.J. Copeland, A.R Liddle and M. Trodden, Phys. Rev. D68 (2003) 023512 |astro-ph/0304277|.

14. L. Susskind, |hep-th/0302219|.

15. S. B. Giddings, S. Kachru and J. Polchinski, Phys. Rev. D66, 106006 (2002).

16. S. Kachru, R. Kallosh, A. Linde and S. P. Trivedi, Phys. Rev. D68 (2003) 046005 |hep-th/0301240|.

17. S.W. Hawking, in the proceedings of the Nuffield Workshop 1981:0423 (QC178:W6:1981); P.C.W. Davies, Prog. Part. Nucl. Phys. 10 (1983) 1; S. Weinberg, Phys. Rev. Lett. 59 (1987) 2607).

18. K.G. Wilson and J.B. Kogut, Phys. Rept. 12 (1974) 75.

19. S. Weinberg, Physica A96 (1979) 327; for a more recent short summary, see: C.P. Burgess, in Barcelona 1998, Radiative corrections: Application of quantum field theory to phenomenology 471-488, |hep-ph/9812470|.

20. G. 't Hooft, in Cargese Summer Inst. 1979:135 (QCD161:S77:1979) (reprinted in 't Hooft, G. (ed.): Under the spell of the gauge principle 352-374, and in Farhi, E. (ed.), Jackiw, R. (ed.): Dynamical gauge symmetry breaking 345-367).

21. B. Zumino, Nucl. Phys. B89 (1975) 535.

22. S. Weinberg, Phys. Rev. Lett. 18 (1967) 188-191; Phys. Rev. D13 (1976) 974-996; L. Susskind, Phys. Rev. D20 (1979) 2619-2625; for a more recent review see C.P. Burgess, Phys. Rep. C330 (2000) 193 |hep-th/9808176|.

23. J. Frieman, C. Hill, A. Stebbins and I. Waga Phys. Rev. Lett. 752077 (1995) |astro-ph/9505060|; D. Cormier and R. Holman, Phys. Rev. Lett. 84 (2000) 5936 |hep-ph/0001168|.

24. S. Dimopoulos and S. Thomas, in Phys. Lett. B573 (2003) 13-19 |hep-th/0307004|.

25. A. Albrecht, C.P. Burgess, F. Ravndal and C. Skordis, Phys. Rev. D65 (2002) 123506 |hep-th/0105261|.

26. Early examples include: A.D. Dolgov, in the proceedings of the Nuffield Workshop 1981:0449 (QC178:W6:1981); F. Wilczek, Phys. Rept. 104 (1984) 143; R.D. Peccei, J. Sola and C. Wetterich, Phys. Lett. B195 (1987) 183; S.M. Barr and D. Hochberg, Phys. Lett. B211 (1988) 49.

27. Y. Aghababaie, C.P. Burgess, S. Parameswaran and F. Quevedo, Nucl. Phys. B680 (2004) 389-414, |hep-th/0304256|.

28. Y. Aghabababie, C.P. Burgess, J.M. Cline, H. Firouzjahi, S. Parameswaran, F. Quevedo, G. Tasinato and I. Zavala, JHEP 0309 (2003) 037 (48 pages) |hep-th/0308064|.

29. C.P. Burgess, Ann. Phys. 313 (2004) 283-401 |hep-th/0402200|.

30. C.P. Burgess, J. Matias and F. Quevedo, |hep-ph/0404135|.

31. C.P. Burgess, F. Quevedo, G. Tasinato and I. Zavala, |hep-th/0408109|. 
32. D. Atwood, C.P. Burgess, E. Filotas, F. Leblond, D. London and I. Maksymyk, Phys. Rev. D63 (2001) 025007 |hep-ph/0007178|.

33. G. Azuelos, P.H. Beauchemin and C.P. Burgess, |hep-ph/0401125|; P.H. Beauchemin, G. Azuelos and C.P. Burgess, J. Phys. G30 (2004) N17 |hep-ph/0407196|.

34. S. Cullen and M. Perelstein, Phys. Rev. Lett. 83 (1999) 268 |hep-ph/9903422|; V. Barger, T. Han, C. Kao, R.-J. Zhang, Phys. Lett. B461 (1999) 34-42, |hep-ph/9905474|; C. Hanhart, D.R. Phillips, S. Reddy, M.J. Savage, Nucl. Phys. B595 (2001) 335 |nucl-th/0007016|; J.A. Pons, D.R. Phillips and S. Reddy, Phys. Lett. B509 (2001) 1-9, |astro-ph/0102063|.

35. S. Hannestad and G. Raffelt, Phys. Rev. Lett. 87 (2001) 051301, |hep-ph/0103201|; Phys. Rev. Lett. 88 (2002) 071301, |hep-ph/0110067|; Phys. Rev. D67 (2003) 125008, Erratum-ibid. D69 (2004) 029901, |hep-ph/0304029|; S. Hannestad, Phys. Rev. D64 (2001) 023515, |hep-ph/0102290|.

36. N. Arkani-Hamed, S. Dimopoulos and G. Dvali, Phys. Lett. B429 (1998) 263 |hep-ph/9803315|; Phys. Rev. D59 (1999) 086004 |hep-ph/9807344|.

37. I. Antoniadis, N. Arkani-Hamed, S. Dimopoulos and G. Dvali, Phys. Lett. B436 (1998) 257 |hep-ph/9804398|; P. Horava and E. Witten, Nucl. Phys. B475 (1996) 94 |hep-th/9603142|; Nucl. Phys. B460 (1996) 506 |hep-th/9510209|; E. Witten, Nucl. Phys. B471 (1996) 135 |hep-th/9602070|; J. Lykken, Phys. Rev. D54 (1996) 3693 |hep-th/9603133|; I. Antoniadis, Phys. Lett. B246 (1990) 377.

38. For a review with references see J. Polchinski, TASI Lectures on D-Branes [hep-th/9611050|.

39. C. D. Hoyle, U. Schmidt, B. R. Heckel, E. G. Adelberger, J. H. Gundlach, D. J. Kapner, H. E. Swanson, Phys. Rev. Lett. 86 (2001) 1418-1421, |hep-ph/0011014|; For a recent review with references, see E.G. Adelberger, B.R. Heckel and A.E. Nelson, Ann. Rev. Nucl. Part. Sci. 53 (2003) 77-121, |hep-ph/0307284|.

40. E. Kiritsis and C. Kounnas, Nucl. Phys. B503 (1997) 117 [hep-th/9703059]; T. Banks and M. Dine, Nucl. Phys. B479 (1996) 173 [hep-th/9605136]; E. Dudas and C. Grojean, Nucl. Phys. B507 (1997) 553 [hep-th/9704177]; I. Antoniadis and M. Quirós, Phys. Lett. B416 (1998) 327 [hep-th/9707208]; Z. Lalak and S. Thomas, Nucl. Phys. B515 (1998) 55 [hep-th/9707223]; E. Dudas, Phys. Lett. B416 (1998) 309 [hep-th/9709043]; H. P. Nilles, M. Olechowski and M. Yamaguchi, Phys. Lett. B415 (1997) 24 [hep-th/9707143]; T. Li, J. Lopez and D. Nanopoulos, Mod. Phys. Lett. A12 (1997) 2647 [hep-ph/9702237]; K. Choi, Phys. Rev. D56 (1997) 6588 [hep-th/9706171]; K. Choi, H. B. Kim and C. Muñoz, Phys. Rev. D57 (1998) 7521 [hep-th/9711158]; A. Lukas, B. Ovrut and D. Waldram, Phys. Rev. D57 (1998) 7529 [hep-th/9711197]; Nucl. Phys. B532 (1998) 43 [hep-th/9710208]; E. Mirabelli and M. Peskin, Phys. Rev. D58 (1998) 065002 [hep-th/97065002]; K. Benakli, Phys. Rev. D60, 104002 (1999) [hep-ph/9809582]; C. P. Burgess, L. E. Ibáñez and F. Quevedo, Phys. Lett. B447, 257 (1999) [hep-ph/9810535]; L. Randall and R. Sundrum, Nucl. Phys. B557 (1999) 79, [hep-th/9810155]; A. Anisimov, M. Dine, M. Graesser and S. Thomas, [hep-th/0201256].

41. H. Nishino and E. Sezgin, Phys. Lett. 144B (1984) 187; Nucl. Phys. B278 (1986) 353; S. Randjbar-Daemi, A. Salam, E. Sezgin and J. Strathdee, Phys. Lett. B151 (1985) 351; A. Salam and E. Sezgin, Phys. Lett. B 147 (1984) 47.

42. L.J. Romans, Nucl. Phys. B269 (1986) 691-711.

43. S. Samuel and J. Wess, Nucl. Phys. B221 (1983) 153; J. Bagger and J. Wess, Phys. Lett. B138 (1984) 105; J. Hughes, J. Liu and J. Polchinski, Phys. Lett. B180 (1986) 370; J. Hughes and J. Polchinski, Nucl. Phys. B278 (1986) 147; J. Bagger and A. Galperin, Phys. Lett. B336 (1994) 25 [hep-th/9406217]; Phys. Rev. D55 (1997) 1091 [hep-th/9608177]; I. Antoniadis, H. Partouche and T. R. Taylor, Phys. Lett. B372 (1996) 83 [hep-th/9512006]; S. Ferrara, L. Girardello and M. Poratti, Phys. Lett. B376 (1996) 275 [hep-th/9512180]; J. Bagger, Nucl. Phys. Proc. Suppl. 52A (1997) 362 [hep-th/9610022]; R. Altendorfer and J. Bagger, in Particles, strings and cosmology, Boston (1998) 742 |hep-th/9809171|; Phys. Lett. B460 (1999) 127 |hep-th/9904213|; J. Louis, [hep-th/0203138].

44. J. M. Cornwall, D. N. Levin and G. Tiktopoulos, Phys. Rev. D10, 1145 (1974) [Erratum-ibid. D 11, 972 (1975)]; M. S. Chanowitz, M. Golden and H. Georgi, Phys. Rev. D36, 1490 (1987); M. S. Chanowitz, Ann. Rev. Nucl. Part. Sci. 38, 323 (1988); C. P. Burgess and D. London, Phys. Rev. Lett. 69 (1992) 3428; Phys. Rev. D48 (1993) 4337 [hep-ph/9203216].

45. E. Dudas and J. Mourad, Phys. Lett. B514 (2001) 173 [hep-th/0012071]; J. Bagger, F. Feruglio and F. Zwirner, JHEP 0202 (2002) 010 [hep-th/0108010]; I. Antoniadis, K. Benakli and A. Laugier, [hep-th/0111209]; K. A. Meissner, H. P. Nilles and M. Olechowski, [hep-th/0205166]; M. Klein, [hep-th/0205300]; C.P. Burgess, E. Filotas, M. Klein and F. Quevedo, JHEP 0310 (2003) 041 (29 pages) |hep-th/0209190]; I. Antoniadis and M. Tuckmantel, Nucl. Phys. B697 (2004) 3-47 [hep-th/0406010|.

46. A. Vilenkin, Phys. Rev. D23 (1981) 852.

47. J.-W. Chen, M.A. Luty and E. Pontón, JHEP 0009 (2000) 012, |hep-th/0003067|.

48. N. Arkani-Hamed, S. Dimopoulos, N. Kaloper and R. Sundrum, Phys. Lett. B 480 (2000) 193, |hep-th/0001197|; S. Kachru, M. B. Schulz and E. Silverstein, Phys. Rev. D 62 (2000) 045021, |hep-th/0001206|.

49. S. Forste, Z. Lalak, S. Lavignac and H. P. Nilles, Phys. Lett. B 481 (2000) 360, hep-th/0002164, JHEP 0009 (2000) 034, |hep-th/0006139|;

C. Csaki, J. Erlich, C. Grojean and T.J. Hollowood, Nucl. Phys. $B 584$ (2000) 359-386, |hep-th/0004133|;

C. Csaki, J. Erlich and C. Grojean, Nucl. Phys. B604 (2001) 312-342, |hep-th/0012143|;

J.M. Cline and H. Firouzjahi, Phys. Rev. D65 (2002) 043501, |hep-th/0107198|.

50. G.W. Gibbons, R. Güven and C.N. Pope, |hep-th/0307238|.

51. S. Randjbar-Daemi and V. Rubakov, |hep-th/0407176|; H. M. Lee and A. Papazoglou, |hep-th/0407208|; V.P. Nair and S. Randjbar-Daemi, |hep-th/0408063|.

52. G. Gibbons and C. Pope, |hep-th/0307052|.

53. S. M. Carroll and M. M. Guica, |hep-th/0302067|; I. Navarro, JCAP 0309 (2003) 004 |hep-th/0302129|. 
54. A. G. Cohen and D. B. Kaplan, Phys. Lett. B215, 67 (1988); A. Vilenkin and P. Shellard, "Cosmic Strings and other Topological Defects," Cambridge University Press (1994).

55. R. Gregory, Phys. Rev. Lett. 59 (1987) 740.

56. R. Gregory and C. Santos, Phys. Rev. D56, 1194 (1997) |gr-qc/9701014|.

57. J. Garriga and M. Porrati, |hep-th/0406158|.

58. J. Vinet et. al., in preparation.

59. I. Navarro, Class. Quant. Grav. 20 (2003) 3603 |hep-th/0305014|; H. P. Nilles, A. Papazoglou and G. Tasinato, Nucl. Phys. B 677 (2004) 405 |hep-th/0309042|; J. Vinet and J. M. Cline, |hep-th/0406141|; M. L. Graesser, J. E. Kile and P. Wang, |hep-th/0403074|.

60. Y. Aghababaie, C.P. Burgess, S. Parameswaran and F. Quevedo, JHEP 0303 (2003) 032, |hep-th/0212091|.

61. D. Hoover et. al, in preparation.

62. D. Ghilencea et. al., in preparation.

63. P.B. Gilkey, J. Diff. Geom. 10 (1975) 601; Adv. Math. 15 (1975) 334; S. Christensen, Ph.D. thesis, University of Texas, 1977; B. Dewitt, in Les Houches 1983, Proceedings, Relativity, Groups and Topology, 381-738.

64. J.S. Dowker, Phys. Rev. D36 (1987) 3095; D. Kabat, |hep-th/9503016|; D.V. Fursaev and S.N. Solodukhin, |hep-th/9501127|; L. De Nardo, D.V. Fursaev and G. Miele, |hep-th/9610011|; D.V. Fursaev and G. Miele, |hep-th/9605153|;

65. E. Elizalde, K. Kirsten and Y. Kubyshin, Z. Phys. C70 (1996) 159 |hep-th/9410101|; A. Albrecht, C.P. Burgess, F. Ravndal and C. Skordis, Phys. Rev. D65 (2002) 123506 |hep-th/0105261|; I. Antoniadis, K. Benakli and M. Quiros, New J. Phys. 3 (2001) 20 |hep-th/0108005|; M. Ito, Nucl. Phys. B668 (2003) 322 |hep-ph/0301168|; S. Matsuda and S. Seki, |hep-th/0404121|.

66. P. Candelas and S. Weinberg, Nucl. Phys. B237 (1984) 397; R. Kantowski and K.A. Milton, Phys. Rev. D35 (1987) 549 ; D. Birmingham, R. Kantowski and K.A. Milton, Phys. Rev. D38 (1988) 1809.

67. D.V. Fursaev, Phys. Lett. B334 (1994) 53; L. De Nardo, D.V. Fursaev and G. Miele, |hep-th/9610011|; P. Chang and J.S. Dowker, |hep-th/9210013]; E. Ponton and E. Poppitz, JHEP 0106 (2001) 019 |hep-ph/0105021|; J.S. Dowker and K. Kirsten, |hep-th/0205029|.

68. J. Kerimo and H. Lu, Phys. Lett. B576 (2003) 219-226 |hep-th/0307222|; M. Cvetic, G.W. Gibbons and C.N. Pope, Nucl. Phys. B677 (2004) 164-180, |hep-th/0308026|.

69. S. Parameswaran et. al., in preparation.

70. A. Albrecht and C. Skordis, Phys. Rev. Lett. 842076 (2000) (astro-ph/9908085).

71. For extant proposals which relate Dark Energy dynamics to earlier inflationar epochs, see: P. J. E. Peebles and A. Vilenkin, Phys. Rev. D59 (1999) 063505 |astro-ph/9810509|; M. Peloso and F. Rosati, JHEP 9912 (1999) 026, |hep-ph/9908271|; A.S. Majumdar, Phys. Rev. D64 (2001) 083503, |astro-ph/0105518|; K. Dimopoulos and J. W. F. Valle, Astropart. Phys. 18 (2002) 287 |astro-ph/0111417|; M. Malquarti and A.R. Liddle, Phys. Rev. D66 (2002) 023524, astro-ph/0203232 ; N.J. Nunes and E.J. Copeland, Phys. Rev. D66 (2002) 043524, |astro-ph/0204115|; M. Giovannini, Phys. Rev. D67 (2003) 123512, |hep-ph/0301264|.

72. N. Arkani-Hamed, L. J. Hall, C. F. Kolda and H. Murayama, Phys. Rev. Lett. 85 (2000) 4434, astro-ph/0005111].

73. E.W. Kolb and R. Slansky, Phys. Lett. B135 (1984) 378; J. Saito, Prog. Theor. Phys. 77 (1987) 322; G. Servant and T.M.P. Tait, Nucl. Phys. $\mathbf{B 6 5 0}$ (2003) 391, |hep-ph/0206071|; H.-C. Cheng, J.L. Feng and K.T. Matchev, Phys. Rev. Lett. 89 (2002) 211301, |hep-ph/0207125|.

74. L.J. Hall and D. Smith, Phys. Rev. D60 (1999) 085008 |hep-ph/9904267|; M. Fairbairn, Phys. Lett. B508 (2001) 335-339|hep-ph/0101131|;

S. Hannestad, Phys. Rev. D64 (2001) 023515 |hep-ph/0102290|.

75. R.N. Mohapatra, S. Nussinov and A. Perez-Lorenzana, Phys. Rev. D68 (2003) $116001 \mid$ hep-ph/0308051|.

76. M. Bourgay, et.al., Nature 426 (2003) 531; A.G. Lyne, et.al., Science 303 (2004) 1153; S.M. Ransom, V.M. Kaspi, R. Ramachandran, P. Demorest, D.C. Backer, E.D. Pfahl, F.D. Ghigo and D.L. Kaplan, astro-ph/0404149|.

77. G.F. Giudice, R. Rattazzi and J.D. Wells, Nucl. Phys. B544 (1999) 3-38, |hep-ph/9811291|; E.A. Mirabelli, M. Perelstein and M.E. Peskin, Phys. Rev. Lett. 82 (1999) 2236-2239, |hep-ph/9811337|; J.L. Hewett, Phys. Rev. Lett. 82 (1999) 4765-4768, |hep-ph/9811356|; T Han, J.D. Lykken and R.-J. Zhang, Phys. Rev. D59 (1999) 105006, |hep-ph/9811350|; I. Antoniadis, K. Benakli and M. Quiros, Phys. Lett. B460 (1999) 176-183, |hep-ph/9905311|; S. Cullen, M. Perelstein and M.E. Peskin, Phys. Rev. D62 (2000) 055012, |hep-ph/0001166|; CDF Collaboration, Phys. Rev. Lett. 89 (2002) 281801, |hep-ex/0205057|.

78. D. Gross, Phys. Lett. B197 (1987) 129; Nucl. Phys. B303 (1988) 407; D. Amati, Phys. Lett. B197 (1987) 81; Int. J. Mod. Phys. A3 (1988) 1615; H. Verlinde and E. Verlinde, Nucl. Phys. B371 (1992) 246-268, |hep-th/9110017|.

79. K.R. Dienes, E. Dudas and T. Gherghetta, Nucl. Phys. B557 (1999) 25; R. N. Mohapatra, S. Nandi and A. Perez-Lorenzana, Phys. Lett. B466, 115 (1999)|hep-ph/9907520|; R. N. Mohapatra and A. Perez-Lorenzana, Nucl. Phys. B576, 466 (2000) |hep-ph/9910474|; R. Barbieri, P. Creminelli and A. Strumia, Nucl. Phys. B585, 28 (2000) |hep-ph/0002199|.

80. J. Matias et. al. in preparation. 